Pacific Journal of Mathematics

REPRÉSENTATIONS MONOMIALES DES GROUPES DE LIE . 


\title{
REPRÉSENTATIONS MONOMIALES DES GROUPES DE LIE NILPOTENTS
}

\author{
HidénORI FuJIWARA
}

\begin{abstract}
On va étudier dans un cadre de la méthode des orbites la formule de Plancherel pour des représentations monomiales d'un groupe de Lie nilpotent et l'expliciter dans le cas de multplicités finies en calculant les coefficients intervenants.
\end{abstract}

0. Introduction. Cette étude a pour origine la thèse de Benoist [2], qui nous suggère d'écrire concrètement la formule de Plancherel abstraite due à Penney [17] pour une représentation monomiale d'un groupe de Lie nilpotent.

Soit $G$ un groupe de Lie nilpotent connexe et simplement connexe d'algèbre de Lie g. D'après la méthode des orbites [13], le dual unitaire $\hat{G}$ de $G$, l'ensemble des classes d'équivalence des représentations unitaires irréductibles de $G$, est paramétré par l'espace $\mathrm{g}^{*} / G$ des orbites de l'action coadjointe de $G$ dans l'espace vectoriel $\mathrm{g}^{*}$ dual de $\mathfrak{g}$. Pour $\pi \in \hat{G}$, on notera $\Omega(\pi)$ l'orbite coadjointe de $G$ associée à $\pi$. Dans ce cadre, on considère une représentation monomiale $\tau=\operatorname{ind}_{H}^{G} \chi$ induite par un caractère unitaire $\chi$ d'un sous-groupe analytique $H$ de $G$. Au fait, h etant la sous-algèbre de Lie correspondant à $H, \chi$ s'écrit sous la forme $\chi(\exp X)=e^{\sqrt{-1} f(X)}(X \in \mathfrak{h})$ avec $f \in \mathfrak{g}^{*}$ telle que $\mathfrak{h}$ soit totalement isotrope pour la forme $B_{f}: B_{f}(X, Y)=f([X, Y])$ quels que soient $X, Y$ dans g. D’après Grélaud [11], la désintégration centrale canonique de $\tau$,

$$
\tau \simeq \int_{\hat{G}}^{\oplus} m(\pi) \pi d \nu(\pi)
$$

s'obtient comme suit, ce qui a été démontré aussi par Corwin et Greenleaf. Quant à la mesure, la classe de $\nu$ est l'image par l'application de Kirillov $\theta: g^{*} \rightarrow \hat{G}$ de celle de la mesure de Lebesgue sur $f+\mathfrak{h}^{\perp}$, où $\mathfrak{h}^{\perp}$ désigne l'orthogonal de $\mathfrak{h}$ dans $\mathfrak{g}^{*}$. En ce qui concerne la fonction $m(\pi)$ de multiplicité, $m(\pi)$ est le nombre de composantes connexes de $\left(f+\mathfrak{h}{ }^{\perp}\right) \cap$ $\Omega(\pi)$ si et seulement si chaque composante est une variété différentielle de dimension égale à $\frac{1}{2} \operatorname{dim} \Omega(\pi)$ pour $\nu$-presque partout, sinon $m(\pi)$ est l'infini pour toute $\pi$. En tout case, $m(\pi)$ est égal au nombre de $H$-orbites inclues dans $\left(f+\mathfrak{h}^{\perp}\right) \cap \Omega(\pi)$ (Duflo, cf. [11]). 
On note $\mathscr{H}_{\tau}$ l'espace de $\tau, \mathscr{H}_{\tau}^{\infty}$ celui des vecteurs $C^{\infty}$ de $\tau$ et $\mathscr{H}_{\tau}^{-\infty}$ l'antidual de $\mathscr{H}_{\tau}^{\infty}$, c'est-à-dire l'espace vectoriel des formes antilinéaires continues de $\mathscr{H}_{\tau}^{\infty}$ dans $\mathbf{C}$, muni de la topologie de dual fort. En notant $e$ l'élément neutre de $G$ et posant $a_{\tau}(\phi)=\overline{\phi(e)}$ quel que soit $\phi \in \mathscr{H}_{\tau}^{\infty}$, on obtient un élément cyclique $a_{\tau}$ de $\mathscr{H}_{\tau}^{-\infty}$.

Le but de cette étude sera d'établir la formule de Plancherel concrète pour la représentation cyclique $\left(\tau, a_{\tau}\right)$ lorsque $\tau$ est de multiplicités finies, ce qui veut dire d'expliciter d'une façon particulière pour elles la formule de Plancherel abstraite due à Penney [17].

Je tiens à remercier Yves Benoist, Gérard Lion, Bernard Magneron et Michel Duflo de leur intérêt pour ce travail.

1. Notations et rappels. Soit $G$ un groupe de Lie nilpotent connexe et simplement connexe d'algèbre de Lie g. En notant exp l'application exponentielle, on écrira $G=\operatorname{expg}$. Soient $V, W$ des espaces vectoriels réels de dimension finie tels que $W \subset V$. On note $V^{*}$ l'espace vectoriel dual de $V$ et $W^{\perp, V^{*}}$, ou plus simplement $W^{\perp}$ si cela ne prête pas à confusion, l'orthogonal de $W$ dans $V^{*}$. Etant donnée $f \in g^{*}$, le noyau de la forme bilinéaire $B_{f}$ se note $\mathfrak{g}(f): \mathfrak{g}(f)=\left\{X \in \mathfrak{g} ; B_{f}(X, Y)=0\right.$ pour tout $Y \in \mathfrak{g}\}$. On dit qu'une sous-algèbre de $\mathrm{g}$ est subordonnée à $f$ si elle est totalement isotrope pour $B_{f}$. On note $S(f, \mathrm{~g})$ l'ensemble de telles sous-algèbres et $M(f, g)$ celui des sous-algèbres qui sont en même temps des sous-espaces totalement isotropes maximals. Un élément de $M(f, \mathfrak{g})$ sera appelé polarisation réelle au point $f$. Enfin, soit $Q(f, \mathfrak{g})$ l'ensemble des sous-algèbres $\mathfrak{h}$ telles que $\mathfrak{h}+g(f)$ soit un sous-espace totalement isotrope maximal.

Soit $\mathfrak{h} \in S(f, \mathfrak{g})$. Donnons-nous un caractère $\chi_{f}$ du sous-groupe analytique $H=\exp \mathfrak{h}$ correspondant à $\mathfrak{h}$ par, quel que soit $X \in \mathfrak{h}$, $\chi_{f}(\exp X)=e^{\sqrt{-1} f(X)}$. On fabrique la représentation induite $\tau=$ $\rho(f, \mathfrak{h}, G)=\operatorname{ind}_{H}^{G} \chi_{f}$ de $G$. Par définition même d'une représentation induite, $\tau$ se réalise par translations à gauche dans l'espace de Hilbert $\mathscr{H}_{\tau}$ des fonctions $\phi$ sur $G$ vérifiant $\phi(g h)=\chi_{f}(h)^{-1} \phi(g)$ quels que soient $g \in G$ et $h \in H$, et de carré intégrable sur $G / H$ pour une mesure invariante. On appelle monomiale une représentation induite comme celle-ci par un caractère unitaire d'un sous-groupe fermé.

Soit $\bar{\theta}: g^{*} / G \rightarrow \hat{G}$ la bijection de Kirillov induite $\operatorname{par} \theta$. Pour $\pi \in \hat{G}$, on notera $\Omega(\pi)$ l'orbite correspondante: $\Omega(\pi)=\bar{\theta}^{-1}(\pi)$. On munit $\mathrm{g}^{*} / G$ et $\hat{G}$ de leurs structures topologiques et boréliennes habituelles. Il est bien connu que $\bar{\theta}$ est un isomorphisme borélien, même un homéomorphisme, de $g^{*} / G$ sur $\hat{G}$ (cf. [10]). On confondra parfois les classes d'équivalence dans $\hat{G}$ avec leurs représentantes, et la relation d'équivalence entre deux représentations $\pi_{1}, \pi_{2}$ se notera $\pi_{1} \simeq \pi_{2}$ ou même $\pi_{1}=\pi_{2}$. 
Lorsque g décrit $G$, on notera $d_{G}(g)$ ou $d g$ une mesure de Haar sur $G$ et $d \dot{g}$ une mesure invariante sur un espace homogène de $G$. Soit $\pi$ une représentation unitaire (continue) de $G$ dans un espace de Hilbert (séparable) $\mathscr{H}_{\pi}$. Notons pour $a \in \mathscr{H}_{\pi}^{ \pm \infty}$ et $b \in \mathscr{H}_{\pi}^{\mp \infty},\langle a, b\rangle$ l'image de $b$ par $a$. Soit $\mathscr{D}(G)$ l'espace des fonctions $C^{\infty}$ sur $G$ à valeurs complexes et à support compact. Pour $\phi \in \mathscr{D}(G)$, en faisant le choix d'une $d g$, on pose

$$
\pi(\phi)=\int_{G} \phi(g) \pi(g) d g
$$

et remarqu'alors que $\pi(\phi) \mathscr{H}_{\pi}^{-\infty} \subset \mathscr{H}_{\pi}^{\infty}$ quelle que soit $\phi \in \mathscr{D}(G)$ (cf. [7]).

Soient $H$ un sous-groupe fermé de $G$ et $\chi$ un caractère de $H$. On note $\left(\mathscr{H}_{\pi}^{-\infty}\right)^{H, \chi}$ le sous-espace vectoriel des éléments a de $\mathscr{H}_{\pi}^{-\infty}$ vérifiant $\pi(h) a=\chi(h) a$ pour tout $h \in H$.

Comme plus haut, soient $f \in \mathfrak{g}^{*}, \mathfrak{h} \in S(f, \mathfrak{g})$ et $\tau=\rho(f, \mathfrak{h}, G)=$ ind $_{H}^{G} \chi_{f}$ avec $H=\exp \mathfrak{h}, \chi_{f}(\exp X)=e^{i f(X)}, i=\sqrt{-1}$, pour $X \in \mathfrak{h}$. On s'intéressera à la formule de Plancherel abstraite, due à Penney [17] et à Bonnet [5], appliquée à la représentation cyclique $\left(\tau, a_{\tau}\right)$, où $a_{\tau} \in$ $\left(\mathscr{H}_{\tau}^{-\infty}\right)^{H, x_{f}}$ est donné, pour $\phi$ dans $\mathscr{H}_{\tau}^{\infty}$, ce qui entraîne que $\phi$ est une fonction $C^{\infty}$ sur $G$ (cf. [18]), par $a_{\tau}(\phi)=\overline{\phi(e)}$.

Lorsque $\mathfrak{h}$ appartient à $M(f, \mathfrak{g}), \tau$ est irréductible et l'on connaît le résultat dû à Howe [12] disant que, pour $\pi \in \hat{G}$, l'espace $\left(\mathscr{H}_{\pi}^{-\infty}\right)^{H, \chi_{f}}$ est égal à $\mathbf{C} a_{\pi}$ si $\pi=\tau$ et trivial si $\pi \neq \tau$. Si $\mathfrak{h} \notin M(f, \mathfrak{g})$, on désintègre $\left(\tau, a_{\tau}\right)$ en désintégration centrale canonique:

$$
\tau=\int_{\hat{G}}^{\oplus} m(\pi) \pi d \nu(\pi), \quad a_{\tau}=\int_{\hat{G}}^{\oplus} a_{\pi} d \nu(\pi) .
$$

D'après l'unicité de désintégration [17], les $a_{\pi}$ étant $\nu$-presque partout dans $\left(m(\pi) \mathscr{H}_{\pi}^{-\infty}\right)^{H, \chi_{f}}$, on a $a_{\pi}=\left(a_{\pi}^{k}\right)_{1 \leq k \leq m(\pi)}$ avec $a_{\pi}^{k} \in\left(\mathscr{H}_{\pi}^{-\infty}\right)^{H, \chi_{f}}$ et, pour $\phi \in \mathscr{D}(G)$,

$$
\left\langle\tau(\phi) a_{\tau}, a_{\tau}\right\rangle=\int_{\hat{G}} \sum_{k=1}^{m(\pi)}\left\langle\pi(\phi) a_{\pi}^{k}, a_{\pi}^{k}\right\rangle d \nu(\pi) \quad \text { (cf. [5]). }
$$

On choisit maintenant une mesure de Haar $d h$ sur $H$ et utilise dans $\mathscr{H}_{\tau}$ la norme définie au moyen de la mesure quotient $d \dot{g}=d g / d h$ sur l'espace homogène $G / H$. Formons, pour $\phi \in \mathscr{D}(G)$, un élément $\phi_{H}^{f}$ de $\mathscr{H}_{\tau}^{\infty}$ par la formule

$$
\phi_{H}^{f}(g)=\int_{H} \phi(g h) \chi_{f}(h) d h \quad(g \in G) .
$$

Alors, le membre gauche de l'égalité (1) n'est autre que la moyenne $\phi_{H}^{f}(e)$ de $\phi$ sur $H$ pour poids $\chi_{f}$. 
En effet, pour $\psi$ dans $\mathscr{H}_{\tau}$, en posant $\phi^{*}(g)=\overline{\phi\left(g^{-1}\right)}(g \in G)$, il vient

$$
\begin{aligned}
\left\langle\tau(\phi) a_{\tau}, \psi\right\rangle & =\left\langle a_{\tau}, \tau\left(\phi^{*}\right) \psi\right\rangle=\int_{G} \phi\left(g^{-1}\right) \overline{\psi\left(g^{-1}\right)} d g \\
& =\int_{G} \phi(g) \overline{\psi(g)} d g=\int_{G / H} \overline{\psi(g)} d \dot{g} \int_{H} \phi(g h) \chi_{f}(h) d h .
\end{aligned}
$$

D'où

$$
\begin{aligned}
\tau(\phi) a_{\tau} & =\phi_{H}^{f}, \\
\left\langle\tau(\phi) a_{\tau}, a_{\tau}\right\rangle & =\left\langle\overline{a_{\tau}, \tau(\phi) a_{\tau}}\right\rangle=\phi_{H}^{f}(e) .
\end{aligned}
$$

On récrit ainsi la formule de Plancherel abstraite pour la représentation cylcique monomiale $\left(\tau, a_{\tau}\right)$ :

ThEORÈme 1 ([5], [17]). La désintégration centrale canonique de $\tau=$ $\rho(f, \mathfrak{h}, G)$ se notant

$$
\tau=\int_{\hat{G}}^{\oplus} m(\pi) \pi d \nu(\pi)
$$

il existe dans $\left(\mathscr{H}_{\pi}^{-\infty}\right)^{H, \chi_{f}}$, pour toute $\pi \in \hat{G}$, des éléments $a_{\pi}^{k}, 1 \leq k \leq$ $m(\pi)$, avec lesquels la formule

$$
\phi_{H}^{f}(e)=\int_{\hat{G}} \sum_{k=1}^{m(\pi)}\left\langle\pi(\phi) a_{\pi}^{k}, a_{\pi}^{k}\right\rangle d \nu(\pi)
$$

s'établit pour n'importe quelle $\phi$ dans $\mathscr{D}(G)$.

Comme Benoist [2], [3], nous pratiquerons dans des cas particuliers un calcul explicite de $\left(a_{\pi}^{k}\right)_{\pi \in \hat{G}, 1 \leq k \leq m(\pi)}$ pour obtenir une formule de Plancherel concrète.

Pour terminer cette section on va examiner de près un exemple.

EXEMPLE 1. Soit $\mathfrak{g}$ algèbre de Lie nilpotente de dimension 4 définie sur la base $\left(e_{1}, e_{2}, e_{3}, e_{4}\right)$ par les crochets: $\left[e_{1}, e_{2}\right]=e_{3},\left[e_{1}, e_{3}\right]=e_{4}$. Soit $f=e_{4}^{*}$ relativement à la base duale de $\mathrm{g}^{*}$ et prenons $\mathfrak{h}=\mathbf{R} e_{2} \oplus \mathbf{R} e_{4}$ dans $S(f, \mathfrak{g})$. On considère $f_{\lambda}=\lambda e_{3}^{*}+e_{4}^{*}(\lambda \in \mathbf{R})$ dans $f+\mathfrak{h}^{\perp}$ et trouve alors $\mathfrak{g}\left(f_{\lambda}\right)=\mathbf{R}\left(e_{2}-\lambda e_{3}\right) \oplus \mathbf{R} e_{4}$. Donc, $\mathfrak{h} \in Q\left(f_{\lambda}, \mathfrak{g}\right)$ pour $\lambda$ non nul. Soit $G=\exp \mathfrak{g}$. Pour $l \in f+\mathfrak{h}^{\perp}$ vérifiant $l\left(e_{3}\right) \neq 0$, l'intersection $\left(f+\mathfrak{h}^{\perp}\right) \cap G \cdot l$ consiste en deux droites dont chacune est une $H$-orbite pour $H=\exp \mathfrak{h}$, tandis qu'elle est une droite contenant des $H$-orbites en nombre infini pour $l \in f+\mathfrak{h}^{\perp}$ s'annulant en $e_{3}$. On voit aussitôt que 
$p\left(f+\mathfrak{h}^{\perp}\right)=\left\{G \cdot f_{\lambda} ; \lambda \geq 0\right\}, p$ étant l'application canonique de $g^{*}$ sur l'espace $g^{*} / G$ des orbites, et qu'en posant $\pi_{\lambda}=\bar{\theta}\left(G \cdot f_{\lambda}\right)$ avec l'application de Kirillov $\bar{\theta}: \mathfrak{g}^{*} / G \rightarrow \hat{G}$,

$$
\rho(f, \mathfrak{h}, G)=\operatorname{ind}_{H}^{G} \chi_{f} \simeq 2 \int_{0}^{\infty} \pi_{\lambda} d \lambda,
$$

ce qui est aussi traité dans [11].

En prenant une polarisation réelle $\mathfrak{f}=\mathbf{R} e_{2} \oplus \mathbf{R} e_{3} \oplus \mathbf{R} e_{4}$ au point $f_{\lambda}$ et en réalisant $\pi_{\lambda} \in \hat{G}$ dans $L^{2}(\mathbf{R})$ sous l'identification $\mathscr{H}_{\pi_{\lambda}} \ni \phi \leftrightarrow \Phi \in$ $L^{2}(\mathbf{R})$ donnée par $\Phi(t)=\phi\left(\exp t e_{1}\right)(t \in \mathbf{R})$, on constate que l'espace $\mathscr{H}_{\pi_{\lambda}}^{\infty}$ se réalise comme espace de Schwartz $\mathscr{S}(\mathbf{R})$ et par suite que $\left(\mathscr{H}_{\pi_{\lambda}}^{-\infty}\right)^{H, \chi_{f}}=\mathbf{C} a_{\lambda}^{1} \oplus \mathbf{C} a_{\lambda}^{2}$ où $a_{\lambda}^{1}: \Phi \mapsto \overline{\Phi(0)}$ tandis que $a_{\lambda}^{2}: \Phi \mapsto \overline{\Phi(2 \lambda)}$ si $\lambda \neq 0$ et $\Phi \mapsto \overline{(d \Phi / d t)(0)}$ si $\lambda=0$. Il vient ainsi que $\operatorname{dim}\left(\mathscr{H}_{\pi_{\lambda}}^{-\infty}\right)^{H, \chi_{f}}=2$, égale à la multiplicité en $\tau$. De même, $\left(\mathscr{H}_{\pi}^{-\infty}\right)^{H, \chi_{f}}=\{0\}$ si $\pi$ n'est équivalente à aucune $\pi_{\lambda}$.

Quant à la formule de Plancherel, on pose, pour $\psi \in \mathscr{D}(G)$,

$$
\psi_{H}^{f}(g)=\int_{H} \psi(g h) \chi_{f}(h) d h \quad(g \in G),
$$

où $d h=d x_{2} d x_{4}$ si $h=\exp x_{2} e_{2} \exp x_{4} e_{4}$. Choisissons $d g=\prod_{j=1}^{4} d x_{j}$ pour $g=\prod_{j=1}^{4} \exp x_{j} e_{j}$. Dans ces circonstances, on a la formule:

$$
\psi_{H}^{f}(e)=\frac{1}{2 \pi} \int_{0}^{\infty}\left\{\left\langle\pi_{\lambda}(\psi) a_{\lambda}^{1}, a_{\lambda}^{1}\right\rangle+\left\langle\pi_{\lambda}(\psi) a_{\lambda}^{2}, a_{\lambda}^{2}\right\rangle\right\} d \lambda
$$

En effet, soient $K=\exp \mathfrak{f}^{k}$ et $d k=\prod_{j=2}^{4} d x_{j}$ pour $k=\prod_{j=2}^{4} \exp x_{j} e_{j}$. Alors, $\Psi_{\lambda}^{1}=\pi_{\lambda}(\psi) a_{\lambda}^{1} \in \mathscr{H}_{\pi_{\lambda}}^{\infty}=\mathscr{S}(\mathbf{R})$ s'obtient par

$$
\Psi_{\lambda}^{1}(t)=\int_{K} \psi\left(\exp t e_{1} \cdot k\right) \chi_{f_{\lambda}}(k) d k \quad(t \in \mathbf{R}),
$$

et par suite

$$
\left\langle\pi_{\lambda}(\psi) a_{\lambda}^{1}, a_{\lambda}^{1}\right\rangle=\int_{K} \psi(x) \chi_{f_{\lambda}}(k) d k .
$$

De même, $\Psi_{\lambda}^{2}=\pi_{\lambda}(\psi) a_{\lambda}^{2} \in \mathscr{S}(\mathbf{R})$ s'obtient par

$$
\Psi_{\lambda}^{2}(t)=\int_{K} \psi\left(\exp (t-2 \lambda) e_{1} \cdot k\right) \chi_{f_{-\lambda}}(k) d k \quad(t \in \mathbf{R})
$$

et puis

$$
\left\langle\pi_{\lambda}(\psi) a_{\lambda}^{2}, a_{\lambda}^{2}\right\rangle=\int_{K} \psi(k) \chi_{f_{-\lambda}}(k) d k
$$


De tout ce qui précède,

$$
\begin{aligned}
& \frac{1}{2 \pi} \int_{0}^{\infty}\left\{\left\langle\pi_{\lambda}(\psi) a_{\lambda}^{1}, a_{\lambda}^{1}\right\rangle+\left\langle\pi_{\lambda}(\psi) a_{\lambda}^{2}, a_{\lambda}^{2}\right\rangle\right\} d \lambda \\
& \quad=\frac{1}{2 \pi} \int_{0}^{\infty} d \lambda \int_{\mathbf{R}} d x_{3} \int_{H}\left(e^{i \lambda x_{3}}+e^{-i \lambda x_{3}}\right) \psi\left(h \exp x_{3} e_{3}\right) \chi_{f_{\lambda}}(h) d h \\
& \quad=\frac{1}{2 \pi} \int_{\mathbf{R}^{2}} e^{i \lambda x_{3}} d \lambda d x_{3} \int_{H} \psi\left(h \exp x_{3} e_{3}\right) \chi_{f}(h) d h \\
& \quad=\int_{H} \psi(h) \chi_{f}(h) d h=\psi_{H}^{f}(e)
\end{aligned}
$$

à l'aide de la formule de Plancherel pour $\mathbf{R}$.

2. Représentations monomiales, multiplicités. Dans tout ce qui suit, sauf mention contraire, $G$ désigne un groupe de Lie nilpotent connexe et simplement connexe d'algèbre de Lie g. Supposons que $G$ agit par une action unipotente dans un espace vectoriel réel $V$ de dimension finie. Soit $v$ un vecteur $G$-invariant non nul dans $V$. Quand on pose, pour $x$ arbitrairement fixé dans $V, L_{x}=x+\mathbf{R} v$, il se produit deux possibilités: soit $L_{x} \cap G \cdot x=\{x\}$, soit $L_{x} \cap G \cdot x=L_{x}$. En d'autres termes, la droite $L_{x}$ rencontre l'orbite $G \cdot x$ en un seul point, ou bien $y$ est complètement contenue. Selon ces deux possibilités, qui ne dépendent que de l'orbite, une orbite sera dite respectivement non-saturée ou saturée (cf. [10], [19], [20]). Soient $l \in V^{*}$ et $W$ un sous-espace vectoriel de $V$. Notons $X$ le sous-espace affine $l+W^{\perp}$ de $V^{*}$. Or $G$ agit dans $V^{*}$ par l'action contragrédiente et introduit dans $X$ une relation d'équivalence, dont l'espace quotient se note $Y=X / G$ et dont une classe sera encore appelée par abus de language $G$-orbite.

Soient $\mu$ une mesure positive finie sur $X$ équivalente à la mesure de Lebesgue et $\tilde{\mu}$ son image par la projection $p: X \rightarrow Y$. En voyant que $Y$ est un espace borélien analytique (cf. [1], [4]), on désintègre $\mu$ relativement à $\tilde{\mu}$ : pour tout $y \in Y$ on trouve une mesure $\mu_{y}$ sur $X$, concentrée sur $p^{-1}(y)$ et telle que

$$
\mu(E)=\int_{Y} \mu_{y}(E) d \tilde{\mu}(y)
$$

pour tout sous-ensemble borélien $E$ de $X$. La famille $\left\{\mu_{y}\right\}_{y \in Y}$ est unique à un ensemble $\tilde{\mu}$-négligeable près.

LEMME 1. Le support de $\mu_{y}$ est $p^{-1}(y)$ tout entier pour $\tilde{\mu}$-presque tout $y \in Y$. 
Démonstration. Posons $\tilde{Y}=\left\{y \in Y\right.$; $\left.\operatorname{supp} \mu_{y} \neq p^{-1}(y)\right\}$ est montrons $\tilde{\mu}(\tilde{Y})=0$ par récurrence sur $\operatorname{dim} V$. On prend un sous-espace $G$-invariant $V_{0}$ de codimension 1 dans $V$. On suppose d'abord $W \subset V_{0}$ et exprime par indice 0 des objets correspondant à $V_{0}: X_{0}=l_{0}+W^{\perp, V_{0}^{*}}$ avec $l_{0}=l \mid V_{0}$ $V_{0}^{*}=\left(V_{0}\right)^{*}$ etc. Par hypothèse de récurrence $\tilde{\mu}_{0}\left(\tilde{Y}_{0}\right)=0$. Si on prend un élément $l \in V^{*}$ s'annulant sur $V_{0}, l$ est évidemment $G$-invariant. Soit $X_{1}$ (resp. $X_{2}$ ) l'ensemble des éléments de $X$ dont l'orbite sous $G$ soit saturée (resp. non-saturée) par rapport à $l$. On sait alors que $X_{1}$ ou $X_{2}$ est $\mu$-négligeable (cf. [10], [20]).

Lorsque $\mu\left(X_{1}\right)=0$, on peut reproduire les raisonnements faits dans les pages 33 à 38 de [10]. En voici une esquisse: l'espace $Y$ (resp. $\tilde{Y}$ ) s'identifie à $Y_{0} \times \mathbf{R}$ (resp. $\left.\tilde{Y}_{0} \times \mathbf{R}\right)$ a un ensemble négligeable près et, sous cette identification, $\tilde{\mu}$ est équivalente au produit de $\tilde{\mu}_{0}$ par la mesure de Lebesgue sur R. Ainsi, $\tilde{\mu}(\tilde{Y})=0$ s'ensuit de $\tilde{\mu}_{0}\left(\tilde{Y}_{0}\right)=0$.

Lorsque $\mu\left(X_{2}\right)=0$, on a $Y=Y_{0}$ à un ensemble négligeable près et $\tilde{\mu}$ est équivalente à $\tilde{\mu}_{0}$. De plus, pour $\tilde{\mu}$-presque tout $y \in Y, p^{-1}(y)=$ $p_{0}^{-1}(y) \times \mathbf{R}$ et $\mu_{y}$ est équivalente au produit de $\left(\mu_{0}\right)_{y}$ par la mesure de Lebesgue sur R. Donc, $\tilde{Y}=\tilde{Y}_{0}$ et $\tilde{\mu}_{0}\left(\tilde{Y}_{0}\right)=0$ entraîne $\tilde{\mu}(\tilde{Y})=0$.

Supposons $W \not \subset V_{0}$. On fixe un élément $w$ dans $W$ qui n'appartient pas à $V_{0}: V=\mathbf{R} w \oplus V_{0}$ et $W=\mathbf{R} w \oplus\left(W \cap V_{0}\right)$. On pose $W_{0}=W \cap V_{0}$, $X_{0}=l_{0}+\left(W_{0}\right)^{\perp, V_{0}^{*}} \subset V_{0}^{*}$ et identifie cette fois $V_{0}^{*}$ au sous-espace $\left\{v^{*} \in\right.$ $\left.V^{*} ; v^{*}(w)=l(w)\right\}$ de $V^{*}$. Alors, $X, \mu, Y, \tilde{\mu}$ et $\tilde{Y}$ s'identifient respectivement à $X_{0}, \mu_{0}, Y_{0}, \tilde{\mu}_{0}$ et à $\tilde{Y}_{0}$, d'où le résultat.

Maintenant, comme dans la section précédente, soient $f \in \mathfrak{g}^{*}, \mathfrak{h} \in$ $S(f, \mathfrak{g}), \chi_{f}$ un caractère unitaire de $H=\exp \mathfrak{h}$ défini par $\chi_{f}(\exp X)=$ $e^{i f(X)}$ pour $X \in \mathfrak{h}$ et $\tau=\rho(f, \mathfrak{h}, G)=\operatorname{ind}_{H}^{G} \chi_{f}$. Soit

$$
\tau=\int_{\hat{G}}^{\oplus} m(\pi) \pi d \nu(\pi)
$$

la décomposition centrale canonique de $\tau$. Lorsqu'on note $\mu$ une mesure finie sur $\mathrm{g}^{*}$ équivalente à la mesure de Lebesgue sur $X=f+\mathfrak{h}^{\perp}, p$ la projection de $\mathfrak{g}^{*}$ sur l'espace $\mathfrak{g}^{*} / G$ et $\theta=\theta_{G}: \mathfrak{g}^{*} \rightarrow \hat{G}, \bar{\theta}=\bar{\theta}_{G}: \mathfrak{g}^{*} / G \rightarrow$ $\hat{G}$ les applications de Kirillov, $\nu$ s'obtient comme image de $\mu$ par $\theta$ : $\nu=\theta_{*}(\mu)=(\bar{\mu} \circ p)_{*}(\mu)$. On désintègre $\mu$ relativement à $\nu$ : symboliquement,

$$
\mu=\int_{\hat{G}} \mu_{y} d \mu(y),
$$

où $\mu_{y}$ est une mesure sur $X$ portée par l'orbite $(\theta \mid X)^{-1}(y)=X \cap \Omega(y)$. Du Lemme 1, il vient: 
Corollaire 1. Le support de $\mu_{y}$ est l'orbite $X \cap \Omega(y)$ tout entier pour $\nu$-presque tout y dans $\hat{G}$.

On prend dans $g$ un idéal $g_{0}$ de codimension 1 tel que $g_{0}$ contienne $\mathfrak{h}$, et un élément $l \in \mathfrak{g}^{*} \mathrm{~s}^{\prime}$ annulant sur $\mathfrak{g}_{0}$, donc invariant par $G$. On sait que toutes les orbites sont du même type pour $l$ à un ensemble $p_{*}(\mu)$ négligeable près. En posant $G_{0}=\exp \mathfrak{g}_{0}$ et $p r: \mathrm{g}^{*} \rightarrow \mathrm{g}^{*}{ }_{0}$ la projection canonique, on rappelle des résultats bien connus [13], [19]. Si $\Omega$ est une orbite saturée, $\operatorname{pr}(\Omega)$ se compose d'une famille à un paramètre $\left\{\omega_{t}\right\}_{t \in \mathbf{R}}$ de $G_{0}$-orbites, $\operatorname{dim} \Omega=\operatorname{dim} \omega_{t}+2$ et $\bar{\theta}_{G}(\Omega)=\operatorname{ind}_{G_{0}}^{G} \bar{\theta}_{G_{0}}\left(\omega_{t}\right)$ pour tout $t \in \mathbf{R}$, tandis que, si $\Omega$ est non-saturée, $\omega=\operatorname{pr}(\Omega)$ est une $G_{0}$-orbite, $p r$ donne un difféomorphisme de $\Omega$ sur $\omega, p^{-1}(\omega)$ se compose d'une famille à un paramètre $\left\{\Omega_{t}\right\}_{t \in \mathbf{R}}$ de $G$-orbites et que

$$
\operatorname{ind}_{G_{0}}^{G} \bar{\theta}_{G_{0}}(\omega)=\int_{\mathbf{R}}^{\oplus} \bar{\theta}_{G}\left(\Omega_{t}\right) d t
$$

Ces situations ainsi que les notations seront utilisées dans la suite à maintes reprises.

Pour une orbite $\Omega \in \mathrm{g}^{*} / G$, l'intersection $X \cap \Omega, X=f+\mathfrak{h}^{\perp}$ comme plus haut, est une variété algébrique réelle (cf. [19]), ce qui entraîne que le nombre de ses composantes connexes est fini d'après Whitney [21]. Soient $\Xi$ l'ensemble des éléments $l$ dans $X$ tels que $\mathfrak{h} \in Q(l, \mathfrak{g})$, et $Z\left(f+\mathfrak{h}^{\perp}\right)$ celui de tels $l \in X$ que $\operatorname{dim} g(l)$ soit minimum lorsque $l$ décrit $X$. Alors $Z\left(f+\mathfrak{h}^{\perp}\right) \cap \Xi$ est, supposé non vide, un ouvert de Zariski dans $X$ et donc admet dans $X$ le complémentaire $\mu$-négligeable. Dans la condition à remplir pour que $l$ appartienne à $\Xi$, c'est $\operatorname{pr}(l)$ qui compte, autrement dit $p^{-1}(p r(l)) \subset \Xi$ si $l \in \Xi$.

LEMME 2. (i) Soient $\Omega \in \mathrm{g}^{*} / G$ et $C$ une composante connexe de $\left(f+\mathfrak{h}^{\perp}\right) \cap \Omega$. Si C est une H-orbite, alors $\operatorname{dim} C=\frac{1}{2} \operatorname{dim} \Omega$ et $\mathfrak{h} \in Q(l, \mathfrak{g})$ pour $l \in C$ aribitraire.

(ii) Soit $l \in \Xi$. Chaque composante connexe de $\Xi \cap G \cdot l$ est une $H$-orbite de dimension $\frac{1}{2} \operatorname{dim} G \cdot l$.

Démonstration. On garde les notations précédentes, par exemple $X_{0}=$ $f_{0}+\mathfrak{h}^{\perp, \mathfrak{g}_{0}^{*}}$ etc, et adopte la récurrence comme d'habitude. Montrons (i). Soit $l_{0}=\operatorname{pr}(l) \in \mathrm{g}_{0}^{*}$. Si $\Omega$ est non-saturée, la composante connexe $\operatorname{pr}(C)$ de $X_{0} \cap \operatorname{pr}(\Omega)$ est une $H$-orbite. Donc, $\operatorname{dim} \operatorname{pr}(C)=\frac{1}{2} \operatorname{dim} \operatorname{pr}(\Omega)$ et $\mathfrak{h} \in Q\left(l_{0}, \mathfrak{g}_{0}\right)$ d'après l'hypothèse de récurrence. Il en vient aussitôt les résultats cherchés. Supposons $\Omega$ saturée. Il est immédiat que, la composante connexe $\operatorname{pr}(C)$ de $X_{0} \cap \operatorname{pr}(\Omega)$ étant une $H$-orbite, elle représente 
une composante connexe de $X_{0} \cap G_{0} \cdot l_{0}$. De là, $\operatorname{dim} \operatorname{pr}(C)=\frac{1}{2} \operatorname{dim} G_{0} \cdot l_{0}$ et $\mathfrak{h} \in Q\left(l_{0}, \mathfrak{g}_{0}\right)$. Notre hypothèse implique aussi que $\mathfrak{g}(l) \cap \mathfrak{h}$ est de codimension 1 dans $\mathfrak{g}_{0}\left(l_{0}\right) \cap \mathfrak{h}$. On voit ainsi que $\operatorname{dim} C=\frac{1}{2} \operatorname{dim} \Omega$, $\operatorname{dim}(\mathfrak{h}+\mathfrak{g}(l))=\operatorname{dim}\left(\mathfrak{h}+\mathfrak{g}_{0}\left(l_{0}\right)\right)$. La dernière égalité veut dire que $\mathfrak{h} \in$ $Q(l, \mathfrak{g})$.

On s'engage à l'assertion (ii). On définit $\Xi_{0}$ pour $g_{0}$ comme $\Xi$ pour g. Soit $G \cdot l$ non-saturée. Puisque $\mathfrak{h} \in Q\left(l_{0}, \mathfrak{g}_{0}\right)$, chaque composante connexe de $\Xi_{0} \cap G_{0} \cdot l_{0}$ est une $H$-orbite de dimension $\frac{1}{2} \operatorname{dim} G_{0} \cdot l_{0}$ et il suffit de remarquer l'inclusion $\operatorname{pr}(\Xi) \subset \Xi_{0}$.

Supposons $G \cdot l$ saturée. Puisque $\mathfrak{h}+\mathfrak{g}(l)$ est un sous-espace de $\mathfrak{g}_{0}$ totalement isotrope maximal pour $B_{l_{0}}, \mathfrak{h}+\mathfrak{g}(l) \supset \mathfrak{g}_{0}\left(l_{0}\right)$ et par conséquent $\mathfrak{h}+\mathfrak{g}(l)=\mathfrak{h}+\mathfrak{g}_{0}\left(l_{0}\right)$. On en déduit que $\mathrm{pr}^{-1}\left(l_{0}\right) \supset H \cdot l$. Comme $\operatorname{pr}(\Xi) \subset \Xi_{0}$, l'hypothèse de récurrence assure que chaque composante connexe de $\Xi_{0} \cap \Omega_{0}, \Omega_{0}=G_{0} \cdot l_{0}$ est une $H$-orbite de dimension $\frac{1}{2} \operatorname{dim} \Omega_{0}$. Considérons l'action de $G$ dans $\mathfrak{g}_{0}^{*}$.

On choisit une base $\left(X_{1}, \ldots, X_{k}\right)$ supplémentaire (cf. [14]) de $\mathfrak{g}\left(l_{0}\right)=$ $\mathfrak{g}_{0}\left(l_{0}\right)$ dans $\mathfrak{g}$ de manière à ce que le premier élément $X_{1}$ n'appartienne pas à $g_{0}$. Pour $\mathbf{t}=\left(t_{1}, \ldots, t_{k}\right) \in \mathbf{R}^{k}$, on pose $g(\mathbf{t})=\Pi_{j=1}^{k} \exp t_{j} X_{j}$. Il est possible d'identifier $G \cdot l_{0}=\operatorname{pr}(G \cdot l)$ à $\mathbf{R}^{k}$ sous le difféomorphisme $\Phi$ donné par:

$$
\Phi: \mathbf{R}^{k} \ni \mathbf{t}=\left(t_{1}, \ldots, t_{k}\right) \mapsto g(\mathbf{t}) \cdot l_{0}=\left(\prod_{j=1}^{k} \exp t_{j} X_{j}\right) \cdot l_{0} \in G \cdot l_{0} .
$$

On introduit un ouvert $M$ de $\mathbf{R}^{k}$ :

$$
M=\left\{\mathbf{t} \in \mathbf{R}^{k} ; \operatorname{dim}\left(\mathfrak{h}+\mathfrak{g}_{0}\left(g(\mathbf{t}) \cdot l_{0}\right)\right) \geq \operatorname{dim}\left(\mathfrak{h}+\mathfrak{g}_{0}\left(l_{0}\right)\right)\right\},
$$

sur lequel la condition pour que $g(\mathbf{t}) \cdot l_{0}$ appartienne à $X_{0}$ s'écrit

$$
F_{j}(\mathbf{t})=F_{j}\left(t_{1}, \ldots, t_{k}\right)=0
$$

pour certains polynômes $F_{j}, 1 \leq j \leq d=\operatorname{dim} \mathfrak{h}$. Sur $M \cap \Phi^{-1}\left(X_{0}\right)$, le rang de la matrice

$$
\left(\frac{\partial F_{m}}{\partial t_{n}}\right)_{\substack{1 \leq m \leq d \\ 2 \leq n \leq k}}
$$

reste constant égal à $\operatorname{dim} \mathfrak{h}-\operatorname{dim} \mathfrak{g}_{0}\left(l_{0}\right) \cap \mathfrak{h}=\frac{1}{2} \operatorname{dim} \Omega_{0}$ et d'ailleurs le vecteur $\mathbf{v}_{1}=\left(\partial F_{1} / \partial t_{1}, \ldots, \partial F_{d} / \partial t_{1}\right)$ est linéairement indépendant des vecteurs $\mathbf{v}_{j}=\left(\partial F_{1} / \partial t_{j}, \ldots, \partial F_{d} / \partial t_{j}\right), 2 \leq j \leq k$. Il en résulte que la matrice

$$
\left(\frac{\partial F_{m}}{\partial t_{n}}\right)_{1 \leq m \leq d}
$$


possède sur $M \cap \Phi^{-1}\left(X_{0}\right)$ le rang constant égal à $\frac{1}{2} \operatorname{dim} \Omega_{0}+1$, ce qui entraîne que $M \cap \Phi^{-1}\left(X_{0}\right)$ est une sous-variété différentiable de $M$ dont la dimension est $k-\left(\frac{1}{2} \operatorname{dim} \Omega_{0}+1\right)=\frac{1}{2} \operatorname{dim} \Omega_{0}$. Ceci posé, chaque composante connexe de $X_{0} \cap G \cdot l_{0}$ est une $H$-orbite, ce qui nous conduit à l'assertion (ii).

Il a été prévu [20] que, pour tout $l \in Z\left(f+\mathfrak{h}^{\perp}\right)$, les composantes connexes de $\left(f+\mathfrak{h}^{\perp}\right) \cap G \cdot l$ fussent des variétés différentiables, ce que contredit le:

EXEMPLE 2. Soit $\mathfrak{g}$ l'algèbre de Lie nilpotente de dimension 8 définie sur la base $\left(T_{i}, X_{j}\right)(1 \leq i \leq 3,1 \leq j \leq 5)$ par les crochets: $\left[T_{1}, X_{1}\right]=X_{2}$, $\left[T_{2}, X_{1}\right]=X_{3},\left[T_{3}, X_{1}\right]=-X_{4},\left[T_{1}, X_{2}\right]=\left[T_{2}, X_{3}\right]=\left[T_{3}, X_{4}\right]=X_{5}$. Puisque le centre de $g$ est $\mathbf{R} X_{5}$, $\operatorname{dim} g(l) \geq 2$ pour $l \in \mathrm{g}^{*}$ arbitraire. Posons $G=\exp \mathfrak{g}$ et $f=X_{5}^{*} \in \mathfrak{g}^{*}$ par rapport à la base duale de $\mathrm{g}^{*}$. Un calcul direct donne $\mathrm{g}(f)=\mathbf{R} X_{1} \oplus \mathbf{R} X_{5}$ et

$$
G \cdot f=\sum_{i=1}^{3} \mathbf{R} T_{i}^{*}+\frac{1}{2}\left(x_{2}^{2}+x_{3}^{2}-x_{4}^{2}\right) X_{1}^{*}+\sum_{j=2}^{4} x_{j} X_{j}^{*}+X_{5}^{*},
$$

$x_{j} \in \mathbf{R}$ pour $2 \leq j \leq 4$. D'où $\operatorname{dim} G \cdot f=6$ et $\operatorname{dim} \mathfrak{g}(f)$ est minimum. Si l'on prend $\mathfrak{h}=\mathfrak{g}(f)=\mathbf{R} X_{1} \oplus \mathbf{R} X_{5} \in S(f, \mathfrak{g})$, alors $f \in Z\left(f+\mathfrak{h}^{\perp}\right)$ et

$$
\begin{aligned}
\left(f+\mathfrak{h}^{\perp}\right) & \cap G \cdot f \\
= & \left\{l \in \sum_{i=1}^{3} \mathbf{R} T_{i}^{*}+\sum_{j=2}^{4} x_{j} X_{j}+X_{5}^{*} \subset \mathfrak{g}^{*} ; x_{4}^{2}=x_{2}^{2}+x_{3}^{2}\right\},
\end{aligned}
$$

qui est une variété algébrique mais non différentiable.

EXEMPLE 3. Supposons que $X=f+\mathfrak{h}^{\perp}$ admet un élément $l$ dont l'orbite correspond à une représentation de carré intégrable modulo le centre de $G$. On sait [16] que $g(l)$ coïncide avec le centre $z$ de $g$ et que $G \cdot l=l+z^{\perp}$. Donc, $X \cap G \cdot l=l+(\mathfrak{h}+\mathfrak{z})^{\perp}$. D'où dire que $\operatorname{dim} X$ $\cap G \cdot l=\frac{1}{2} \operatorname{dim} G \cdot l$ est équivalent à dire que $\mathfrak{t}=\mathfrak{h}+\mathfrak{z}$ appartient à $M(l, \mathfrak{g})$, et si c'est le cas, on a $H \cdot l=X \cap G \cdot l$. On voit ainsi que

$$
\tau=\operatorname{ind}_{H}^{G} \chi_{f}=m \int_{\hat{G}}^{\oplus} \pi d \nu(\pi)
$$

où $m$ est égal à un ou à l'infini, et $m=1$ si et seulement si $\mathfrak{h}$ appartient à $Q(l, \mathfrak{g})$.

Avant de terminer cette section, donnons quelques exemples au delà du cas nilpotent. Pour le moment, $G$ désigne un groupe de Lie résoluble 
exponentiel d'algèbre de Lie $\mathfrak{g}$. La correspondance entre $\hat{G}$ et $\mathfrak{g}^{*} / G$ reste valable (cf. [4]).

EXEMPLE 4. Supposons qu'il existe une suite croissante

$$
\mathfrak{h}=\mathfrak{g}_{0} \subset \mathfrak{g}_{1} \subset \cdots \subset \mathfrak{g}_{n-1} \subset \mathfrak{g}_{n}=\mathfrak{g}, \quad \operatorname{dim} \mathfrak{g}_{j} / \mathfrak{g}_{j-1}=1
$$

de sous-algèbres de $\mathfrak{g}$ telle que $\mathfrak{g}_{j-1}$ est un idéal de $\mathfrak{g}_{j}$. Dans ce cas aussi, la mesure $\nu$ est construite comme dans le cas nilpotent [20]. Si $\left(f+\mathfrak{h}^{\perp}\right)$ $\cap G \cdot l=H \cdot l$ pour presque toute $l \in f+\mathfrak{h}^{\perp}$, on voit facilement que $\tau=\rho(f, \mathfrak{h}, G)$ est sans multiplicité: à savoir

$$
\tau=\int_{\hat{G}}^{\oplus} \pi d \nu(\pi)
$$

EXEMPLE 5. Etant supposé que $\mathfrak{g}=\mathbf{R} T \oplus \mathbf{R} X \oplus \mathbf{R} Y \oplus \mathbf{R} Z:[T, X]$ $=X,[T, Y]=-Y,[X, Y]=Z$, posons $O(\alpha, \beta)=G \cdot f_{\alpha, \beta}(\beta \neq 0)$ où $f_{\alpha, \beta}=\alpha T^{*}+\beta Z^{*}$ par rapport à la base duale de $\mathrm{g}^{*}$. Un calcul simple montre

$$
O(\alpha, \beta)=t T^{*}+x X^{*}+y Y^{*}+\beta Z^{*}
$$

avec des coefficients vérifiant $x y=\beta(\alpha-t)$.

Prenons d'abord $\mathfrak{h}=\mathbf{R} T \oplus \mathbf{R} X$. On a $f_{\alpha_{0}, \beta_{0}}+\mathfrak{h}^{\perp}=\alpha_{0} T^{*}+\mathbf{R} Y^{*}+$ $\mathbf{R} Z^{*}$ et par suite, pour $\beta$ non nul, $\left.\left(f_{\alpha_{0}, \beta_{0}}+\mathfrak{h}\right)^{\perp}\right) \cap(\alpha, \beta)$ est une $H$-orbite $H \cdot f_{\alpha, \beta}=\alpha_{0} T^{*}+\mathbf{R} Y^{*}+\beta Z^{*}$ si $\alpha=\alpha_{0}$ ou vide si $\alpha \neq \alpha_{0}$. Soient $\mathfrak{f}=\mathbf{R} T \oplus \mathbf{R} X \oplus \mathbf{R} Z, K=\exp$ et $\pi(\alpha, \beta) \in \hat{K}$ associée à la $K$-orbite passant $\alpha T^{*}+\beta Z^{*} \in \mathfrak{l}^{*}$. Alors,

$$
\begin{aligned}
\operatorname{ind}_{H}^{G} \chi_{f_{\alpha_{0}, \beta_{0}}} & \simeq \operatorname{ind}_{K}^{G} \operatorname{ind}_{H}^{K} \chi_{f_{\alpha_{0}, \beta_{0}}} \simeq \operatorname{ind}_{K}^{G} \int_{\mathbf{R}} \pi\left(\alpha_{0}, \beta\right) d \beta \\
& \simeq \int_{\mathbf{R}} \theta\left(O\left(\alpha_{0}, \beta\right)\right) d \beta,
\end{aligned}
$$

$\theta$ étant l'application de Bernat de $\mathrm{g}^{*} / G$ sur $\hat{G}$.

Deuxièmement soit $\mathfrak{h}=\mathbf{R} T \oplus \mathbf{R} Z$. Pour $\beta \neq 0,\left(f_{\alpha_{0}, \beta_{0}}+\mathfrak{h}^{\perp}\right) \cap$ $O(\alpha, \beta)$ est vide si $\beta \neq \beta_{0}$ ou égal, si $\beta=\beta_{0}$, à $\alpha_{0} T^{*}+x X^{*}+y Y^{*}+$ $\beta_{0} Z^{*}$ avec $x y=\beta_{0}\left(\alpha-\alpha_{0}\right)$ qui se compose de deux $H$-orbites si $\alpha \neq \alpha_{0}$.

En passant, soit $g_{2}$ l'algèbre de $a x+b: g_{2}=\mathbf{R} e_{1} \oplus \mathbf{R} e_{2}$ avec le crochet $\left[e_{1}, e_{2}\right]=e_{2}$. Il est bien connu que le groupe $G_{2}=\operatorname{exp~g}_{2}$ a deux représentations $\pi_{ \pm}$de dimension infinie correspondant aux orbites $\pm G_{2}$. $e_{2}^{*}$. Soient $f=\alpha e_{1}^{*} \in \mathfrak{g}_{2}^{*}, \mathfrak{h}_{0}=\mathbf{R} e_{1} \in S\left(f, \mathfrak{g}_{2}\right)$ et $H_{0}=\exp \mathfrak{h}_{0}$. On a, pour $l \in f+\mathfrak{h}_{0}^{\perp, g_{2}^{*}}$ quelconque, ind $G_{H_{0}}^{G_{2}} \chi_{f}=\operatorname{ind}_{H_{0}}^{G_{2}} \chi_{l} \simeq \pi_{+} \oplus \pi_{-}$.

En revenant à notre cas, posons $\mathfrak{n}=\mathbf{R} X \oplus \mathbf{R} Y \oplus \mathbf{R} Z$ et $N=\exp \mathfrak{n}$. Le sous-groupe $K$ étant comme ci-dessus, on trouve $K$ isomorphe à 
$G_{2} \times \mathbf{R}$. Notant $\chi_{\beta_{0}}$ le caractère unitaire de $\mathbf{R}$ donné par $\chi_{\beta_{0}}(\alpha)=e^{i \beta_{0} \alpha}$ et $\times$ le produit de Kronecker extérieur,

$$
\begin{aligned}
\operatorname{ind}_{H}^{G} \chi_{f_{\alpha_{0}, \beta_{0}}} & \simeq \operatorname{ind}_{K}^{G} \operatorname{ind}_{H}^{K} \chi_{f_{\alpha_{0}, \beta_{0}}} \simeq \operatorname{ind}_{K}^{G}\left(\left(\pi_{+} \times \chi_{\beta_{0}}\right) \oplus\left(\pi_{-} \times \chi_{\beta_{0}}\right)\right) \\
& \simeq \operatorname{ind}_{H^{\prime}}^{G}\left(\chi_{f_{1}} \oplus \chi_{f_{2}}\right) \simeq \operatorname{ind}_{N}^{G} \operatorname{ind}_{H^{\prime}}^{N}\left(\chi_{f_{1}} \oplus \chi_{f_{2}}\right) \\
& =2 \operatorname{ind}_{N}^{G} \pi_{\beta_{0}} \simeq 2 \int_{\mathbf{R}}^{\oplus} \theta\left(O\left(\alpha, \beta_{0}\right)\right) d \alpha,
\end{aligned}
$$

où $\mathfrak{h}^{\prime}=\mathbf{R} X \oplus \mathbf{R} Z, H^{\prime}=\exp \mathfrak{h}^{\prime}, f_{j}=\gamma_{j} X^{*}+\beta_{0} Z(j=1,2)$ avec $\gamma_{1}>0$, $\gamma_{2}<0$ et $\pi_{\beta_{0}} \in \hat{N}$ liée à la $N$-orbite passant $\beta_{0} Z^{*}$ dans $\mathfrak{n}^{*}$. Ainsi, on voit une fois encore que la multiplicité est donnée par le nombre de $H$-orbites.

On trouvera dans [2], [11] d'autres exemples concernant nos questions.

3. Vecteurs généralisés et semi-invariants. Revenant au cas nilpotent on reprend les notations précédentes: $G=\exp : \mathfrak{g}, \mathfrak{h} \in S(f, \mathfrak{g})$ et $\chi_{f}$ le caractère unitaire de $H=\operatorname{exph}$ défini par $\chi_{f}(\exp X)=e^{i f(X)}$ pour $X \in \mathfrak{h}$. L'objet à étudier dans cette section, c'est l'espace $\left(\mathscr{H}_{\pi}^{-\infty}\right)^{H, \chi_{f}}$ des vecteurs généralisés de $\pi \in \hat{G}$, semi-invariants par $H$ à poids $\chi_{f}$. On prend un $l \in \Omega(\pi)$, y choisit une polarisation réelle $\mathfrak{b} \in M(l, \mathfrak{g})$ et réalise $\pi$ dans l'espace $L^{2}\left(\mathbf{R}^{m}\right), m=\frac{1}{2} \operatorname{dim} \Omega(\pi)$ comme suit. On dira qu'une base supplémentaire $\left(X_{1}, \ldots, X_{m}\right)$ à $\mathfrak{b}$ dans $\mathfrak{g}$ est adaptée, si pour tout $j$, le sous-espace $\sum_{k=j}^{m} \mathbf{R} X_{k} \oplus \mathfrak{b}$ est une sous-algèbre de $\mathrm{g}$. Prenons-en une $\left(X_{1}, \ldots, X_{m}\right)$. On peut (cf. [19]) alors identifier l'espace $G$ avec $\mathbf{R}^{m} \times B$, où $B=\exp \mathfrak{b}$, au moyen du difféomorphisme $\Phi$ donné par

$$
\Phi: \mathbf{R}^{m} \times B \ni\left(\left(x_{1}, \ldots, x_{m}\right), b\right) \mapsto\left(\prod_{j=1}^{m} \exp x_{j} X_{j}\right) \cdot b \in G,
$$

ce qui nous permet d'identifier l'espace homogène $G / B$ à $\mathbf{R}^{m}$, une mesure de Lebesgue $d x=\prod_{j=1}^{m} d x_{j}$ sur $\mathbf{R}^{m}$ à une mesure invariante sur $G / B$ et l'espace $\mathscr{H}_{\pi}^{\infty}$ des vecteurs $C^{\infty}$ de $\pi$ à l'espace de Schwartz $\mathscr{S}\left(\mathbf{R}^{m}\right)$ (cf. [8]). Ceci étant, l'espace $\mathscr{H}_{\pi}^{-\infty}$ des vecteurs généralisés de $\pi$ est antiisomorphe à l'espace $\mathscr{S}\left(\mathbf{R}^{m}\right)^{\prime}$ des distributions tempérées sur $\mathbf{R}^{m}$. On identifiera parfois $\mathbf{R}^{m}$ au sous-ensemble $\Phi\left(\mathbf{R}^{m} \times\{e\}\right)$ de $G$.

Pour étudier l'espace $\left(\mathscr{H}_{\pi}^{-\infty}\right)^{H, \chi_{f}}$, ce sera sa dimension qui nous dirigera dans la suite. Commençons par regarder le cas où l'on pourrait prendre $\mathfrak{b}$ de manière à ce qu'on ait $\mathrm{gHg}^{-1} \subset B$ pour tout $g \in G$, ce qui est le cas en particulier pour toute $l \in[\mathfrak{h}, \mathfrak{h}]^{\perp} \subset \mathfrak{g}^{*}$ si h est un idéal. Pour $a \in \mathscr{H}_{\pi}^{-\infty}$, dire que $a \in\left(\mathscr{H}_{\pi}^{-\infty}\right)^{H, \chi_{f}}$ équivaut à dire que, pour tous $\phi \in$ $\mathscr{H}_{\pi}^{\infty}, g \in G$ et $h \in H$, on a

$$
\left\langle a,\left(\chi_{f}(h)-\chi_{l}\left(g^{-1} h g\right)\right) \phi\right\rangle=0 .
$$


Donc, lorsqu'on regarde $a$ comme distribution sur $\mathbf{R}^{m}$, son support est contenu dans l'ensemble $S \cap \mathbf{R}^{m}$ où $S=\left\{g \in G ; g \cdot l \in f+\mathfrak{h}^{\perp}\right\}$. En particulier, si $\mathfrak{h}$ est un idéal abélien de $\mathfrak{g},\left(\mathscr{H}_{\pi}^{-\infty}\right)^{H, \chi_{f}}=\{0\}$ pour toute $\pi \in \hat{G}$ telle que $\Omega(\pi)$ ne rencontre pas $f+\mathfrak{h}^{\perp}$.

A supposer que $\mathfrak{h}$ soit un idéal et que $l$ appartienne à $\left(f+\mathfrak{h}^{\perp}\right) \cap$ $\Omega(\pi)$, on choisit $\mathfrak{b}$ de sorte qu'elle contienne $\mathfrak{h}$. Si $\mathfrak{h} \notin Q(l, \mathfrak{g})$, on trouve l'inclusion stricte $H \subsetneq B \subsetneq G\left(f^{\prime}\right)$ où $G\left(f^{\prime}\right)$ désigne le stabilisateur dans $G$ de $f^{\prime}=f \mid \mathfrak{h} \in \mathfrak{h}^{*}$ et jouit de la propriété $G\left(f^{\prime}\right) \cdot f=\left(f+\mathfrak{h}^{\perp}\right) \cap G \cdot f$, ce qui entraîne directement $\operatorname{dim}\left(\mathscr{H}_{\pi}^{-\infty}\right)^{H, \chi_{f}}=\infty$, en regardant ses éléments $a_{g}$ fabriqués, pour $g \in G\left(f^{\prime}\right)$, par $a_{g}(\phi)=\overline{\phi(g)}$ quel que soit $\phi \in \mathscr{H}_{\pi}^{\infty}$.

Soit maintenant $\mathfrak{h} \in Q(l, \mathfrak{g})$, c'est-à-dire $\mathfrak{b}=\mathfrak{h}+\mathfrak{g}(l)=\mathfrak{g}\left(f^{\prime}\right)$, le stabilisateur dans $g$ de $f^{\prime} \in \mathfrak{h}^{*}$, et par suite $B=G\left(f^{\prime}\right)$. S'il en est ainsi, le support de $a \in\left(\mathscr{H}_{\pi}^{-\infty}\right)^{H, \chi_{f}}$ regardé comme distribution tempérée sur $\mathbf{R}^{m}$ se réduit à l'origine. Allons constater que $a$ est proportionelle à la mesure de Dirac $\delta$ à l'origine. En effet, introduisant parmi les dérivées de $\delta$ l'ordre lexicographique relativement aux opérateurs $\partial / \partial x_{1}, \partial / \partial x_{2}, \ldots$, $\partial / \partial x_{m}$, ce qui veut dire que

$$
\frac{\partial^{|\alpha|} \boldsymbol{\delta}}{\partial x_{1}^{\alpha_{1}} \cdots \partial x_{m}^{\alpha_{m}}}>\frac{\partial^{|\beta|} \delta}{\partial x_{1}^{\beta_{1}} \cdots \partial x_{m}^{\beta_{m}}} \quad\left(|\alpha|=\sum_{j=1}^{m} \alpha_{j},|\beta|=\sum_{j=1}^{m} \beta_{j}\right)
$$

si et seulement s'il existe un indice $k, 1 \leq k \leq m$, tel que $\alpha_{1}=$ $\beta_{1}, \ldots, \alpha_{k-1}=\beta_{k-1}, \alpha_{k}>\beta_{k}$, soit

$$
c \cdot \frac{\partial^{|\alpha|} \boldsymbol{\delta}}{\partial x_{1}^{\alpha_{1}} \cdots \partial x_{m}^{\alpha_{m}}} \quad(0 \neq c \in \mathbf{C})
$$

le terme dominant de $a$ par cet ordre et supposons que $\alpha_{m} \geq 1$.

Lorsqu'on prend $\phi \in \mathscr{S}\left(\mathbf{R}^{m}\right)$ de la forme $\phi\left(x_{1}, \ldots, x_{m}\right)=\prod_{j=1}^{m} \phi_{j}\left(x_{j}\right)$ avec $\phi_{j} \in \mathscr{S}(\mathbf{R})$ vérifiant

$$
\begin{gathered}
\frac{d^{\alpha_{j}-1} \phi_{j}}{d x_{j}^{\alpha_{j}-1}}(0)=\cdots=\frac{d \phi_{j}}{d x_{j}}(0)=\phi_{j}(0)=0, \\
\frac{d^{\alpha_{j}} \phi_{j}}{d x_{j}^{\alpha_{j}}}(0)=1 \quad(1 \leq j \leq m-1) ; \\
\frac{d^{\alpha_{m}-2} \phi_{m}}{d x_{m}^{\alpha_{m}-2}}(0)=\cdots=\frac{d \phi_{m}}{d x_{m}}(0)=\phi_{m}(0)=0, \quad \frac{d^{\alpha_{m}-1} \phi_{m}}{d x_{m}^{\alpha_{m}}-1}(0)=1,
\end{gathered}
$$

la condition (2) se récrit

$$
\left.\frac{d^{\alpha_{m}}}{d x_{m}^{\alpha_{m}}}\left\{\left(\chi_{f}(h)-\chi_{l}\left(\exp -x_{m} X_{m} \cdot h \cdot \exp x_{m} X_{m}\right)\right) \phi_{m}\left(x_{m}\right)\right\}\right|_{x_{m}=0}=0
$$


quel que soit $h \in H$. Ou encore, $l\left(\left[X_{m}, X\right]\right)=0$ pour n'importe quel $X \in \mathfrak{h}$, ce qui est contradictoire et l'on en conclut que $\alpha_{m}=0$. En répétant ce procédé, on arrive finalement au résultat attendu.

On voit ainsi la:

Proposition 1. Supposons que $\mathfrak{h}$ soit un idéal. Etant donnée $\pi \in \hat{G}$ telle que $\left(f+\mathfrak{h}^{\perp}\right) \cap \Omega(\pi)$ ne soit pas vide, on constate que $\left(\mathscr{H}_{\pi}^{-\infty}\right)^{H, x_{f}}$ est de dimension 1 ou $\infty$ selon que $\mathfrak{h}$ appartient ou $n$ 'appartient pas à $Q(l, \mathfrak{g})$ pour un et donc tout l dans $\left(f+\mathfrak{h}^{\perp}\right) \cap \Omega(\pi)$.

REMARQue 1. Notons ici petits faits divers quelques fois utiles pour déterminer l'espace $\left(\mathscr{H}_{\pi}^{-\infty}\right)^{H, \chi_{f}}$ dans des cas bien particuliers. Soient $\pi \in \hat{G}$ et $C_{1}, \ldots, C_{n}$ les composantes connexes de $\left(f+\mathfrak{h}^{\perp}\right) \cap \Omega(\pi)$. Supposons encore l'existence de $l \in \Omega(\pi)$ et de $\mathfrak{b} \in M(l, \mathfrak{g})$ telles que $\mathrm{gHg}^{-1}$ $\subset B$ pour tout $g \in G$, mais $\mathfrak{h}$ n'est plus supposée être un idéal. Pour $1 \leq j \leq n$, on choisit arbitrairement des éléments $g_{j}$ de $G$ de façon qu'on ait $g_{j} \cdot l \in C_{j}$, et considère $a_{j} \in \mathscr{H}_{\pi}^{-\infty}$ obtenu par $\left\langle a_{j}, \phi\right\rangle=\widehat{\phi\left(g_{j}\right)}$ quel que soit $\phi \in \mathscr{H}_{\pi}^{\infty}$. Il est immédiat que $a_{1}, \ldots, a_{n}$ appartiennent à $\left(\mathscr{H}_{\pi}^{-\infty}\right)^{H, \chi_{f}}$ et sont linéairement indépendants. En effet, le support de $a_{j}$ n'étant autre que la double classe fermée $\mathrm{Hg}_{j} B$, il suffit de montrer que $H g_{j} B \neq H g_{k} B$ si $j \neq k$. Sinon, l'ensemble connexe $\left(f+\mathfrak{h}^{\perp}\right) \cap g_{j}$. $\left(l+\mathfrak{b}^{\perp}\right)$ de $\left(f+\mathfrak{h}^{\perp}\right) \cap \Omega(\pi)$ croiserait en même temps $C_{j}$ et $C_{k}$, ce qui est absurde.

Si $C_{j} \subset \Xi$, alors $C_{j}$ est une $H$-orbite d'après le Lemme 2 et il est clair qu' à un scalaire multiplicatif près, $a_{j}$ ne dépend pas du choix de $g_{j}$. Lorsque $\Xi$ contient toutes les $C_{j}$, l'argument fait en chemin à la Proposition 1 affirme $\left(\mathscr{H}_{\pi}^{-\infty}\right)^{H, \chi_{f}}=\sum_{j=1}^{n} \oplus \mathbf{C} a_{j}$ et, si de plus $\Xi$ rencontre $Z\left(f+\mathfrak{h}^{\perp}\right)$, la dimension $n \operatorname{de}\left(\mathscr{H}_{\pi}^{-\infty}\right)^{H, x_{f}}$ est égale à la multiplicité $m(\pi)$ dans la désintégration de $\tau=\operatorname{ind}_{H}^{G} \chi_{f}$.

Pluse généralement, soit $\mathfrak{h} \in S(f, g)$. Si, sous l'identification habituelle, on pose $S=\left\{g \in \mathbf{R}^{m} ; g \cdot l \in f+\mathfrak{h}^{\perp}, g^{-1} H g \subset B\right\}$, il est évident qu'une fonction $\psi$ sur $\mathbf{R}^{m}$ à croissance lente et ayant son support dans $S$ définit un élément de $\left(\mathscr{H}_{\pi}^{-\infty}\right)^{H, \chi_{f}}$ par la formule:

$$
\mathscr{H}_{\pi}^{\infty}=\mathscr{S}\left(\mathbf{R}^{m}\right) \ni \phi \mapsto \int_{\mathbf{R}^{m}} \psi(x) \overline{\phi(x)} d x,
$$

$d x$ étant une mesure de Lebesgue sur $\mathbf{R}^{m}$.

On reprend son chemin, garde les notations et pose $\mathfrak{g}_{0}=\sum_{j=2}^{m} \oplus \mathbf{R} X_{j}$ $\oplus \mathfrak{b}, G_{0}=\exp g_{0}$. Notons $g^{t}$ le sous-groupe à un paramètre $\exp t X_{1}, t$ décrivant R. Soient $\pi_{0}=$ ind $_{B}^{G_{0}} \chi_{l}, \pi_{t}=g^{t} \cdot \pi_{0}$, c'est-à-dire $\pi_{t}\left(g_{0}\right)=$ $\pi_{0}\left(g^{-t} g_{0} g^{t}\right)$ pour $g_{0} \in G_{0}$, et $f_{0}=f \mid g_{0} \in g_{0}^{*}$. Pour examiner un peu le 
cas où $\mathfrak{h}$ n'est pas contenue dans $\mathfrak{g}_{0}$, après avoir remplacé $X_{1}$ au besoin, supposons $X_{1}$ appartenir à $\mathfrak{h}$. Soit $\lambda=f\left(X_{1}\right)$. Comme distribution tempérée sur $\mathbf{R}^{m}, a \in\left(\mathscr{H}_{\pi}^{-\infty}\right)^{H, \chi_{f}}$ vérifie la relation

$$
\left\langle\phi\left(x_{1}+t, x_{2}, \ldots, x_{m}\right), a\right\rangle=\left\langle e^{-i \lambda t} \phi\left(x_{1}, x_{2}, \ldots, x_{m}\right), a\right\rangle
$$

pour tous $\phi \in \mathscr{S}\left(\mathbf{R}^{m}\right)$ et $t \in \mathbf{R}$. D'où on voit qu'il existe une certaine distribtuion tempérée $a_{0}$ relativement aux variables $\left(x_{2}, \ldots, x_{m}\right)$ telle qu'on ait, pour $\phi \in \mathscr{S}\left(\mathbf{R}^{m}\right)$,

$$
\langle a, \phi\rangle=\int_{\mathbf{R}}\left\langle a_{0}, \phi\left(x_{1}, x_{2}, \ldots, x_{m}\right)\right\rangle e^{-i \lambda x_{1}} d x_{1} .
$$

Comme application de ce procédé, on voit le:

EXemple 6. Supposons $G$ métabélien, ce qui veut dire que $[\mathfrak{g}, \mathfrak{g}]$ est abélienne. Dans la désintégration

$$
\tau=\int_{\hat{G}}^{\oplus} m(\pi) \pi d \nu(\pi)
$$

de $\tau=\operatorname{ind}_{H}^{G} \chi_{f}$, la multiplicité $m(\pi)$ est $\operatorname{dim}\left(\mathscr{H}_{\pi}^{-\infty}\right)^{H, \chi_{f}}$ si $\left(f+\mathfrak{h}^{\perp}\right) \cap$ $\Omega(\pi) \subset \Xi$ presque partout pour la mesure $\nu$. En fait, ce qu'on vient de voir nous mène au cas, où $\mathfrak{h} \subset[\mathfrak{g}, \mathfrak{g}]$, étudié antérieurement dans la Remarque 1 car il existe n'importe où une polarisation réelle contenant $[\mathfrak{g}, \mathfrak{g}]$.

Posons $\mathfrak{h}_{0}=\mathfrak{h} \cap \mathfrak{g}_{0}$ et $H_{0}=\exp \mathfrak{h}_{0}$. D'une manière naturelle, $a_{0}$ donne un élément de $\left(\mathscr{H}_{\pi_{t}}^{-\infty}\right)^{H_{0}, \chi_{f}}$ pour tout $t \in \mathbf{R}$. D'ailleurs la restriction $\sigma=\pi \mid G_{0}$ de $\pi$ à $G_{0}$ se désintègre en

$$
\sigma=\int_{\mathbf{R}}^{\oplus} \pi_{t} d t
$$

à laquelle s'associe la formule (3) qui s'interprète comme désintégration de $a$ :

$$
a=\int_{\mathbf{R}}^{\oplus} a_{0} e^{i \lambda t} d t
$$

Enfin les composantes connexes de $\left(f+\mathfrak{h}^{\perp}\right) \cap \Omega(\pi)$ correspondent par projection pr: $\mathfrak{g}^{*} \rightarrow \mathfrak{g}_{0}^{*}$ aux composantes connexes de $\left(f_{0}+\mathfrak{h}_{0}^{\perp, \mathfrak{g}_{0}^{*}}\right) \cap$ $\Omega_{0}\left(\pi_{0}\right)$.

Cette observation nous permet de retrouver le résultat suivant dû à Howe [12]: pour $\mathfrak{h} \in M(f, \mathfrak{g}), \operatorname{dim}\left(\mathscr{H}_{\pi}^{-\infty}\right)^{H, \chi_{f}}$ est égal à 1 ou 0 selon que $\Omega(\pi)=G \cdot f$ ou non. En effet, soit $l \in \Omega(\pi)$, on se ramène facilement au cas où le centre $z$ de $g$ est de dimension 1 et où $f, l$ ont la même restriction non nulle sur $z$. Prenons un triplet de Heisenberg $(X, Y, Z)$ tel 
que $z=\mathbf{R} Z,[X, Y]=Z, f(Z)=l(Z)=1, f(Y)=0$ et que $g=\mathbf{R} X \oplus$ $\mathfrak{g}_{0}$ avec $\mathfrak{g}_{0}=\{U \in \mathfrak{g} ;[U, Y]=0\}$. Il est possible de choisir une $\mathfrak{b} \in$ $M(l, \mathfrak{g})$ inclue dans $\mathfrak{g}_{0}$. Posons $\lambda=l(Y)$.

Supposons d'abord que $\mathfrak{h}$ n'est pas inclue dans $\mathfrak{g}_{0}$ de sorte qu'on puisse prendre $X$ dans $\mathfrak{h}$. Soit $f^{\prime}=g^{-\lambda} \cdot f_{0} \in g_{0}^{*}$. Il est évident que $\mathfrak{h}^{\prime}=\mathfrak{h}_{0} \oplus \mathbf{R} Y \in M\left(f^{\prime}, \mathfrak{g}_{0}\right)$. De ce qui précède on constate qu'un élément $a \operatorname{de}\left(\mathscr{H}_{\pi}^{-\infty}\right)^{H, \chi_{f}}$ correspond à un certain $a_{0} \in\left(\mathscr{H}_{\pi_{0}}^{-\infty}\right)^{H_{0}, \chi_{f_{0}}}=\left(\mathscr{H}_{\pi_{0}}^{-\infty}\right)^{H^{\prime}, \chi_{f^{\prime}}}$ où $H^{\prime}=\exp \mathfrak{h}^{\prime}$. Compte tenu de ce que les deux orbites $\Omega(\pi)$ et $G \cdot f$ sont saturées, pour obtenir le résultat il suffit d'appliquer l'hypothèse de récurrence.

Supposons ensuite $\mathfrak{h}$ inclue dans $\mathfrak{g}_{0}$. Allons utiliser un sous-groupe à un paramètre $h_{t}=\exp t Y(t \in \mathbf{R})$ appartenant nécessairement à $H \cap B$. Par semi-invariance appliquée à $h_{t}$, un élément $a$ de $\left(\mathscr{H}_{\pi}^{-\infty}\right)^{H, \chi_{f}}$ vérifie, pour tout $t \in \mathbf{R},\left\langle a,\left(1-e^{i(s-\lambda) t}\right) \phi(g)\right\rangle=0$ où $g=\exp s X \cdot g_{0}$ avec $s \in \mathbf{R}, g_{0} \in G_{0}$. Un raisonnement analogue à celui fait dans la démonstration de la Proposition 1 montre qu'il existe un élément $a_{0}$ dans $\mathscr{H}_{\pi_{0}}^{-\infty}=\mathscr{H}_{\pi_{\lambda}}^{-\infty}$ tel qu'on ait, pour $\phi \in \mathscr{H}_{\pi}^{\infty},\langle a, \phi\rangle=\left\langle a_{0}, \tilde{\phi}\right\rangle$, $\tilde{\phi}$ étant donnée par $\tilde{\phi}\left(g_{0}\right)=\phi\left(\exp \lambda X \cdot g_{0}\right), g_{0} \in G_{0}$. Cela implique aussitôt que $a_{0} \in\left(\mathscr{H}_{\pi_{\lambda}}^{-\infty}\right)^{H, \chi_{f_{0}}}$, d'où l'on peut se terminer comme dans le premier cas. Ramarquons que $\lambda$ est une seule valeur de $t \in \mathbf{R}$ satisfaisant à la condition $\exp t X \cdot l \in f+\mathfrak{h}^{\perp}$.

$\mathrm{Eu}$ égard à tout ce qu'on vient de voir, on va modifier légèrement le résultat de Benoist pour associer à chaque composante connexe $C$ de $\left(f+\mathfrak{h}^{\perp}\right) \cap \Omega(\pi)$ inclue dans $\Xi$ un sous-espace de dimension 1 de $\left(\mathscr{H}_{\pi}^{-\infty}\right)^{H, \chi_{f}}$. Etant donée $l \in \Omega(\pi), \pi$ se réalise à l'aide d'une polarisation réelle $\mathfrak{b} \in M(l, \mathfrak{g})$ comme représentation monomiale $\operatorname{ind}_{B}^{G} \chi_{l}$ induite du caractère $\chi_{l}$ de $B=\exp \mathfrak{b}$. Prenons un élément $g \in G$ tel que $g \cdot l \in C$, et une mesure invariante $d \dot{h}$ sur l'espace homogène $H / H \cap \mathrm{gBg}^{-1}$.

Proposition 2. On peut fabriquer un élément non nul a dans $\left(\mathscr{H}_{\pi}^{-\infty}\right)^{H, \chi_{f}}$ par la formule suivante: pour tout $\phi \in \mathscr{H}_{\pi}^{\infty}$,

$$
\langle a, \phi\rangle=\int_{H / H \cap g B g^{-1}} \overline{\phi(g h) \chi_{f}(h)} d \dot{h} .
$$

Démonstration. Voyons d'abord l'intégrale au membre droit est bien définie. En fait, quel que soit $h \in H \cap g B g^{-1}$,

$$
\begin{aligned}
\phi\left(h h^{\prime} g\right) \chi_{f}\left(h h^{\prime}\right) & =\phi\left(h g g^{-1} h^{\prime} g\right) \chi_{f}(h) \chi_{f}\left(h^{\prime}\right) \\
& =\chi_{l}\left(g^{-1} h^{\prime-1} g\right) \phi(h g) \chi_{f}(h) \chi_{f}\left(h^{\prime}\right) \\
& =\chi_{g \cdot l}\left(h^{\prime-1}\right) \chi_{f}\left(h^{\prime}\right) \phi(h g) \chi_{f}(h)=\phi(h g) \chi_{f}(h) .
\end{aligned}
$$


Il existe une base supplémentaire adaptée $\left(X_{1}, \ldots, X_{m}\right)$ à $g \cdot \mathfrak{b}$ dans $\mathfrak{g}$ telle que $\left(X_{i_{1}}, X_{i_{2}}, \ldots, X_{i_{p}}\right), 1 \leq i_{1}<i_{2}<\cdots<i_{p} \leq m$ soit de la même sorte à $\mathfrak{h} \cap \mathfrak{g} \cdot \mathfrak{b}_{\text {dans }} \mathfrak{h}$. Par usage de cette base l'espace translaté de $\mathscr{H}_{\pi}^{\infty}$ par $g$ à droite s'identifie à l'espace de $\operatorname{Sch}$ wartz $\mathscr{S}\left(\mathbf{R}^{m}\right)$ et $d \dot{h}$ à une mesure de Lebesgue sur $\mathbf{R}^{p} \subset \mathbf{R}^{m}$, d'où la continuité de $a$. En réalité cette translation n'est autre qu'un opérateur d'entrelacement entre deux réalisations de $\pi$ aux points $l$ et $g \cdot l$. Enfin un calcul direct assure la semi-invariance nécessaire.

Evidemment, le vecteur généralisé $a$ introduit dans la Proposition 2 ne dépend pas du choix de $g$ à un scalaire multiplicatif près. Il faut maintenant examiner sa dépendance de la réalisation de $\pi$ et c'est ce point-là où s'intervient effectivement l'opérateur d'entrelacement et l'indice de Maslov. Fixons $l$ dans $\Omega(\pi) \cap \Xi$ et considérons deux réalisations de $\pi$ au moyen de deux polarisations $\mathfrak{b}_{1}, \mathfrak{b}_{2} \in M(l, \mathfrak{g}), \pi \simeq \pi_{2}=\operatorname{ind}_{B_{1}}^{G} \chi_{l} \simeq \pi_{2}$ $=\operatorname{ind}_{B_{2}}^{G} \chi_{l}$ où $B_{j}=\exp \mathfrak{b}_{j}(j=1,2)$. On fabrique [14] une isométrie $T_{\mathfrak{b}_{2} \mathfrak{b}_{1}}$ entrelaçant $\pi_{1}$ avec $\pi_{2}$ par la formule, pour tout $\phi \in \mathscr{H}_{\pi_{1}}^{\infty}$,

$$
\left(T_{\mathfrak{b}_{2} \mathfrak{b}_{1}} \phi\right)(g)=\int_{B_{2} / B_{2} \cap B_{1}} \phi(g b) \chi_{l}(b) d \dot{b} \quad(g \in G),
$$

$d \dot{b}$ étant une mesure invariante bien normalisée. L'opérateur $T_{\mathfrak{b}_{2} \mathfrak{b}_{1}}$ se prolonge sur $\mathscr{H}_{\pi_{1}}^{-\infty}$ (cf. [2]) et l'on montre la:

Proposition 3 (cf. [14]). On se donne $a_{1} \in\left(\mathscr{H}_{\pi_{1}}^{-\infty}\right)^{H, \chi_{f}}, a_{2} \in$ $\left(\mathscr{H}_{\pi_{2}}^{-\infty}\right)^{H, \chi_{f}}$ par les formules

$$
\begin{aligned}
\left\langle a_{1}, \phi\right\rangle=\int_{H / H \cap B_{1}} \overline{\phi\left(h_{1}\right) \chi_{f}\left(h_{1}\right)} d \dot{h}_{1}, & \phi \in \mathscr{H}_{\pi_{1}}^{\infty} ; \\
\left\langle a_{2}, \psi\right\rangle=\int_{H / H \cap B_{2}} \overline{\psi\left(h_{2}\right) \chi_{f}\left(h_{2}\right)} d \dot{h}_{2}, & \psi \in \mathscr{H}_{\pi_{2}}^{\infty} .
\end{aligned}
$$

Sous réserve de normalisations convenables des mesures, on obtient

$$
T_{\mathfrak{b}_{2} \mathfrak{b}_{1}} a=e^{(i \pi / 4) \tau\left(\mathfrak{h}+\mathfrak{g}(l), \mathfrak{b}_{2}, \mathfrak{b}_{1}\right)} a_{2} .
$$

où $\tau$ indique l'indice de Maslov [15]. En d'autres termes, quel que soit $\phi \in \mathscr{H}_{\pi_{1}}^{\infty}$,

$$
\begin{aligned}
& \int_{H / H \cap B_{2}} \chi_{f}\left(h_{2}\right) d \dot{h}_{2} \int_{B_{2} / B_{2} \cap B_{1}} \phi\left(h_{2} b\right) \chi_{l}(b) d \dot{b} \\
& =e^{(i \pi / 4) \tau\left(\mathfrak{h}+g(l), \mathfrak{b}_{2}, \mathfrak{b}_{1}\right)} \int_{H / H \cap B_{1}} \phi\left(h_{1}\right) \chi_{f}\left(h_{1}\right) d \dot{h}_{1} .
\end{aligned}
$$


On fixe désormais $l \in \Omega(\pi)$ et $\mathfrak{b} \in M(l, \mathfrak{g})$ de sorte que $\pi=\operatorname{ind}_{B}^{G} \chi_{l}$. Supposons $\Xi$ contenir $\left(f+\mathfrak{h}^{\perp}\right) \cap \Omega(\pi)$ dont les composantes connexes se notent $C_{1}, \ldots, C_{m}$. A chacune d'eux fait correspondre la Proposition 2 un vecteur généralisé: $g_{k}$ étant un élément de $G$ vérifiant $g_{k} \cdot l \in C_{k}$ et $d_{k} \dot{h}$ une mesure invariante sur $H / H \cap g_{k} B g_{k}^{-1}$,

$$
\left\langle a_{\pi}^{k}, \phi\right\rangle=\int_{H / H \cap g_{k} B g_{k}^{-1}} \overline{\phi\left(h g_{k}\right) \chi_{f}(h)} d_{k} \dot{h} \quad(1 \leq k \leq m)
$$

pour tout $\phi \in \mathscr{H}_{\pi}^{\infty}$. On choisit une mesure de Haar $d b=d_{B}(b)$ sur $B$ et définit, pout $\phi \in \mathscr{D}(G)$, une fonction $\tilde{\phi}$ sur $G$ par

$$
\tilde{\phi}(g)=\int_{B} \phi(b g) \chi_{l}(b) d b .
$$

Il est clair que $\tilde{\phi} \in \mathscr{H}_{\pi}^{\infty}$ et un vecteur généralisé $a \in \mathscr{H}_{\pi}^{-\infty}$ donne une distribution $\tilde{a}$ sur $G$ : pour $\phi$ dans $\mathscr{D}(G), \tilde{a}(\phi)=\langle\tilde{\phi}, a\rangle$.

Naturellement le support de la distribution $\tilde{a}_{\pi}^{k}$ coïncide la double classe fermée $H g_{k} B$, ce qui entraîne comme dans la Remarque 1 que $a_{\pi}^{1}, \ldots, a_{\pi}^{m}$ sont linéairement indépendants. D'autre part, soit $a \in$ $\left(\mathscr{H}_{\pi}^{-\infty}\right)^{H, \chi_{f}}$ tel que $\tilde{a}$ soit une distribution sur $H g_{k} B$, autrement dit que $\tilde{a}$ ait $H g_{k} B$ pour son support et son ordre transversal soit nul (cf. [6]). Alors, $a$ est évidemment proportionel à $a_{\pi}^{k}$. Posons $S_{\pi}=\{g \in G ; g$. $\left.\left(l+\mathfrak{b}^{\perp}\right) \cap\left(f+\mathfrak{h}^{\perp}\right) \neq \varnothing\right\}$, qui n'est autre que la réunion disjointe $\sqcup_{k=1}^{m} H g_{k} B$, et désignons par $\left(\mathscr{H}_{\pi}^{-\infty}\right)_{0}^{H, \chi_{f}}$ le sous-espace des $a \in$ $\left(\mathscr{H}_{\pi}^{-\infty}\right)^{H, \chi_{f}}$ tels que ã soit une distribution sur la sous-variété fermée $S_{\pi}$. Concernant la multiplicité on tire de tout ce qui précède la:

Proposition 4. Soit $\tau=\operatorname{ind}_{H}^{G} \chi_{f}$. On se donne la désintégration centrale canonique de $\tau$ :

$$
\tau=\int_{\hat{G}}^{\oplus} m(\pi) \pi d \nu(\pi) .
$$

Supposons $Z\left(f+\mathfrak{h}^{\perp}\right) \cap \Xi$ non vide, autrement dit que $m(\pi)$ sont toutes finies. On peut alors prendre pour multiplicité $m(\pi)$ la dimension de l'espace $\left(\mathscr{H}_{\pi}^{-\infty}\right)_{0}^{H, \chi_{f}}$.

Il serait intéressant d'obtenir un résultat analogue à la proposition dans [9].

4. Formule de Plancherel. On garde les notations précédentes. Soit $\pi \in \hat{G}$ telle que $\Xi$ contienne $\left(f+\mathfrak{h}^{\perp}\right) \cap \Omega(\pi)$ dont les composantes connexes se notent $C_{1}, \ldots, C_{m}$. On fait des choix de $l \in \Omega(\pi), g_{k}(1 \leq k$ $\leq m)$ tels que $g_{k} \cdot l \in C_{k}$ et de plusieurs mesures $d g=d_{G}(g), d h=$ $d_{H}(h), d \dot{g}$ et $d_{k} \dot{h}$ invariantes sur $G, H, G / B$ et $H / H \cap g_{k} B g_{k}^{-1}$. Le choix 
de $d g$ nous donne $\pi=\operatorname{ind}_{B}^{G} \chi_{l}$ et ceux de $d_{1} \dot{h}, \ldots, d_{m} \dot{h}$ les vecteurs généralisés $a_{\pi}^{1}, \ldots, a_{\pi}^{m}$ dans $\left(\mathscr{H}_{\pi}^{-\infty}\right)^{H, \chi_{f}}$ associés aux $C_{1}, \ldots, C_{m}$. On en déduit la mesure quotient $d \ddot{g}$ sur $G / H$ et, grâce à une propriété de transitivité, une mesure $d_{k} \dot{g}$ invariante sur $G / H \cap g_{k} B g_{k}^{-1}$ et son image canonique sur $G / g_{k}^{-1} H g_{k} \cap B$ puis une mesure $d_{k} \dot{b}$ invariante sur $B / B \cap$ $g_{k}^{-1} H g_{k}$. Pour $\phi \in \mathscr{D}(G)$ on forme encore

$$
\phi_{H}^{f}(g)=\int_{H} \phi(g h) \chi_{f}(h) d h \quad(g \in G)
$$

Lemme 3. Pour $\phi \in \mathscr{D}(G)$, on $a$

$$
\left(\pi(\phi) a_{\pi}^{k}\right)(g)=\int_{B / B \cap g_{k}^{-1} H g_{k}} \phi_{H}^{f}\left(g b g_{k}^{-1}\right) \chi_{l}(b) d_{k} \dot{b} \quad(g \in G),
$$

et par suite

(4) $\left\langle\pi(\phi) a_{\pi}^{k}, a_{\pi}^{k}\right\rangle$

$$
=\int_{H / H \cap g_{k} B g_{k}^{-1}} \chi_{f}(h) d_{k} \dot{h} \int_{B / B \cap g_{k}^{-1} H g_{k}} \phi_{H}^{f}\left(h g_{k} b g_{k}^{-1}\right) \chi_{l}(b) d_{k} \dot{b} .
$$

Démonstration. Soit $\psi \in \mathscr{H}_{\pi}^{\infty}$. On calcule:

$$
\begin{aligned}
\left\langle\pi(\phi) a_{\pi}^{k}, \psi\right\rangle & =\int_{G} \phi(g)\left\langle a_{\pi}^{k}, \pi\left(g^{-1}\right) \psi\right\rangle d g \\
& =\int_{G} \phi(g) d g \int_{H / H \cap g_{k} B g_{k}^{-1}} \overline{\psi\left(g h g_{k}\right) \chi_{f}(h)} d_{k} \dot{h} \\
& =\int_{G / H} \phi_{H}^{f}(g) d \ddot{g} \int_{H / H \cap g_{k} B g_{k}^{-1}} \overline{\psi\left(g h g_{k}\right) \chi_{f}(h)} d_{k} \dot{h} \\
& =\int_{G / H} d \ddot{g} \int_{H / H \cap g_{k} B g_{k}^{-1}} \phi_{H}^{f}(g h) \overline{\psi\left(g h g_{k}\right)} d_{k} \dot{h} \\
& =\int_{G / H \cap g_{k} B g_{k}^{-1}} \phi_{H}^{f}(g) \overline{\psi\left(g g_{k}\right)} d_{k} \dot{g} \\
& =\int_{G / B} d \dot{g} \int_{B / B \cap g_{k}^{-1} H g_{k}} \phi_{H}^{f}\left(g b g_{k}^{-1}\right) \overline{\psi(g b)} d_{k} \dot{b} \\
& =\left\langle\int_{B / B \cap g_{k}^{-1} H g_{k}} \phi_{H}^{f}\left(g b g_{k}^{-1}\right) \chi_{l}(b) d_{k} \dot{b}, \psi\right\rangle,
\end{aligned}
$$

d'où le résultat. 
D'après (4), les valeurs $\left\langle\pi(\phi) a_{\pi}^{k}, a_{\pi}^{k}\right\rangle(1 \leq k \leq m)$ ne dépendent pas du choix des $g_{k}$ sous les transformations canoniques de mesures associées, et la Proposition 3 dit que, si l'on change $l \in \Omega(\pi)$ et $\mathfrak{b} \in M(l, \mathfrak{g})$, bref la réalisation de $\pi$, on peut faire choix de mesures $d \dot{g}, d_{k} \dot{h}$ d'une façon à ce qu'elles restent invariantes.

EXEMPLE 7. Si par hasard $\left(f+\mathfrak{h}^{\perp}\right) \cap \Omega(\pi)$ est une seule $H$-orbite, on y prend $l$ et la formule (4) se lit

$$
\left\langle\pi(\phi) a_{\pi}, a_{\pi}\right\rangle=\int_{H / H \cap B} \chi_{f}(h) d \dot{h} \int_{B / B \cap H} \phi_{H}^{f}(h b) \chi_{l}(b) d \dot{b} .
$$

Supposons de plus $\mathfrak{h}$ inclue dans $\mathfrak{b}$ et notons $\mathscr{F} \phi$ la transformée de Fourier de $\phi \circ \exp$ :

$$
(\mathscr{F} \phi)(\xi)=\int_{\mathfrak{g}} e^{i \xi(X)} \phi(\exp X) d X \quad\left(\xi \in \mathfrak{g}^{*}\right),
$$

$d \dot{X}$ étant une mesure de Lebesgue sur g. On a

$$
\begin{aligned}
\left\langle\pi(\phi) a_{\pi}, a_{\pi}\right\rangle & =\int_{B / H} \phi_{H}^{f}(b) \chi_{l}(b) d \dot{b}=\int_{B} \phi(b) \chi_{l}(b) d b \\
& =\int_{B \cdot l}(\mathscr{F} \phi)(\xi) d \xi=\int_{H \cdot l}(\mathscr{F} \phi)(\xi) d \xi
\end{aligned}
$$

avec une mesure de Lebesgue $d \xi$ convenablement normalisée sur $B \cdot l=$ $H \cdot l=l+\mathfrak{b}^{\perp}$.

Utilisant ces coefficients, notre formule de Plancherel concrète va s'écrire. Soeint $\mu$ une mesure de Lebesgue sur $f+\mathfrak{h}^{\perp}$, regardée comme mesure sur $g^{*},[\mu]$ la classe de $\mu$ et $\gamma$ l'image de $[\mu]$ par l'application de Kirillov $\theta: g^{*} \rightarrow \hat{G}$.

THEORÈmE 2. Supposons $Z\left(f+\mathfrak{h}^{\perp}\right) \cap \Xi$ non vide, ce qui veut dire que la représentation monomiale ind ${ }_{H}^{G} \chi_{f}$ est de multiplicités finies. Faisant choix de mesures d $\dot{g}, d_{k} \dot{h}(1 \leq k \leq m(\pi))$ pour $\pi \in \hat{G}$ telles que $\left(f+\mathfrak{h}^{\perp}\right)$ $\cap \Omega(\pi)$ soit non vide et contenu dans $\Xi$, il existe sur $\hat{G}$ une mesure $\nu$ de classe $\gamma$, et ayant la propriété suivante: pour $\phi \in \mathscr{D}(G)$ arbitraire, on a la formule

$$
\phi_{H}^{f}(e)=\int_{\hat{G}} \sum_{k=1}^{m(\pi)}\left\langle\pi(\phi) a_{\pi}^{k}, a_{\pi}^{k}\right\rangle d \nu(\pi) .
$$

Démonstration. Soit $\mathfrak{g}_{0}$ un idéal de codimension 1 dans $g$, et contenant $\mathfrak{h}$. Premièrement, presque toutes les orbites sont supposées non-saturées par rapport à $g_{0}$. Pour presque toute $\pi$, la restriction de $\pi$ à 
$G_{0}=\exp \mathfrak{g}_{0}$ est irréductible, $m(\pi)=m\left(\pi_{0}\right)$ et à un ensemble négligeable près, l'espace borélien $\hat{G}$ s'identifie à $\hat{G}_{0} \times \mathbf{R}$ (cf. [10]). L'hypothèse de récurrence assure l'existence d'une mesure $\nu_{0}$ sur $\hat{G}_{0}$ ayant les propriétés dans l'énoncé du théorème et sous notre identification la mesure $\nu=\nu_{0} \times$ $d x, d x$ étant une mesure de Lebesgue sur $\mathbf{R}$, nous conviendra en posant $a_{\pi}^{k}=a_{\pi_{0}}^{k}$ pour $1 \leq k \leq m(\pi)$.

En effet, soit pr: $\mathrm{g}^{*} \rightarrow \mathrm{g}_{0}^{*}$ l'application canonique. En sorte que $\nu_{0}$ existe, est faisable le choix de $d \dot{g}_{0}, d_{k} \dot{h}_{0}$ et donc de $d_{k} \dot{b}_{0}$, l'indice 0 signifiant des objets correspondants au niveau du sous-group $G_{0}$. On désigne $\mathfrak{b}_{0} \in M\left(l_{0}, \mathfrak{g}_{0}\right)$ la polarisation réelle, ainsi choisie et donnant $\pi_{0}=\theta_{G_{0}}\left(l_{0}\right) \in \hat{G}_{0}$. En se servant de la Proposition 3, on peut supposer que l'application $\hat{G}_{0} \ni \pi_{0} \mapsto l_{0} \in \mathrm{g}_{0}^{*}$ soit la section borélienne $s_{0}$ en Lemme 2.5.5 dans [10]. Pour $l \in \mathrm{g}^{*}$ telle que $\operatorname{pr}(l)=l_{0}$, on peut prendre un élément $T=T\left(l_{0}\right)$, dépendant de $l_{0}$, dans $\mathrm{g}$ de manière qu'on ait $\mathrm{g}(l)=$ $\mathbf{R} T \oplus \mathrm{g}_{0}\left(l_{0}\right)$ et que l'application borélienne $\pi \mapsto\left(\pi \mid G_{0}, l(T)\right)$ donne notre identification entre $\hat{G}$ et $\hat{G}_{0} \times \mathbf{R}$. Or la Proposition 3 nous rend capable de supposer avoir choisi $\mathfrak{b}_{0}$ de façon qu'une polarisation $\mathfrak{b} \in$ $M(l, \mathfrak{g})$ s'obtienne par $\mathbf{R} T \oplus \mathfrak{b}_{0}$ et, en écrivant $b \in B=\exp \mathfrak{b}$ comme $b=\exp t T \cdot b_{0}$ avec $t \in \mathbf{R}$ et $b_{0} \in B_{0}=\exp \mathfrak{b}_{0}$, on constate que $B / B \cap$ $g_{k}^{-1} H g_{k}$ est isomorphe à $\mathbf{R} \times B_{0} / B_{0} \cap g_{k}^{-1} H g_{k}$. On choisit $d \dot{g}$ de sorte que $d_{k} \dot{b}=d t \cdot d_{k} \dot{b}_{0}$.

Dans ces situations, si l'on pose $\lambda=l(T)$, la mesure $\nu=\nu_{0} \times d \lambda / 2 \pi$ nous convient: pour $\phi \in \mathscr{D}(G)$ quelconque, compte tenu de (4) et de l'hypothèse sur $\nu_{0}$,

$$
\begin{aligned}
\int_{\hat{G}} & \sum_{k=1}^{m(\pi)}\left\langle\pi(\phi) a_{\pi}^{k}, a_{\pi}^{k}\right\rangle d \nu(\pi) \\
= & \int_{\hat{G}_{0}} d \nu_{0}\left(\pi_{0}\right) \int_{\mathbf{R}} \frac{d \lambda}{2 \pi} \sum_{k=1}^{m\left(\pi_{0}\right)} \int_{H / H \cap g_{k} B g_{k}^{-1}} \chi_{f}(h) d_{k} \dot{h} \\
& \times \int_{\mathbf{R}} e^{i \lambda t} d t \int_{B_{0} / B_{0} \cap g_{k} H g_{k}^{-1}} \phi_{H}^{f}\left(h g_{k} \exp t T \cdot b_{0} g_{k}^{-1}\right) \chi_{l_{0}}\left(b_{0}\right) d_{k} \dot{b}_{0} \\
= & \int_{\hat{G}_{0}} d \nu_{0}\left(\pi_{0}\right) \sum_{k=1}^{m\left(\pi_{0}\right)} \int_{H / H \cap g_{k} B g_{k}^{-1}} \chi_{f}(h) d_{k} \dot{h} \\
\quad & \times \int_{B_{0} / B_{0} \cap g_{k} H g_{k}^{-1}} \phi_{H}^{f}\left(h g_{k} b_{0} g_{k}^{-1}\right) \chi_{l_{0}}\left(b_{0}\right) d_{k} \dot{b}_{0} \\
= & \int_{\hat{G}_{0}} \sum_{k=1}^{m\left(\pi_{0}\right)}\left\langle\pi_{0}(\phi) a_{\pi_{0}}^{k}, a_{\pi_{0}}^{k}\right\rangle d \nu_{0}\left(\pi_{0}\right)=\phi_{H}^{f}(e)
\end{aligned}
$$

d'après la formule de Plancherel pour $\mathbf{R}$. 
Deuxièmement, supposons que presque toutes les orbites sont saturées. Prenons une $\pi \in \hat{G}$ telle que $\left(f+\mathfrak{h}^{\perp}\right) \cap \Omega(\pi)$ soit non vide et totalement inclu dans $\Xi$. Il existe des représentations irréductibles en nombre fini de $G_{0}$, notées $\pi_{0}^{1}, \ldots, \pi_{0}^{s}$ telles que $\left(f_{0}+\mathfrak{h}^{\perp, g_{0}^{*}}\right) \cap \operatorname{pr}(\Omega(\pi))$ soit la réunion disjointe des $\left(f_{0}+\mathfrak{h}^{\perp, \mathfrak{g}_{0}^{*}}\right) \cap \Omega_{0}\left(\pi_{0}^{j}\right), 1 \leq j \leq s$, dont les composantes connexes se notent $C_{0,1}^{j}, \ldots, C_{0, i_{j}}^{j}$ où $i_{j}=m\left(\pi_{0}^{j}\right)$. Les composantes connexes de $\left(f+\mathfrak{h}^{\perp}\right) \cap \Omega(\pi)$ ne sont autre que $C_{k}^{j}=p^{-1}\left(C_{0, k}^{j}\right)$ pour $1 \leq k \leq m\left(\pi_{0}^{j}\right), 1 \leq j \leq s$. A $C_{0, k}^{j}$ s'associe un $a_{\pi_{0}^{j}}^{k} \in\left(\mathscr{H}_{\pi_{j}^{-\infty}}^{-\infty}\right)^{H, \chi_{f}}$ et à $C_{k}^{j}$ un $a_{\pi}^{r} \in\left(\mathscr{H}_{\pi}^{-\infty}\right)^{H, x_{f}}$ avec $r=\sum_{i=1}^{j-1} m\left(\pi_{0}^{i}\right)+k$.

Par hypothèse de récurrence, ayant choisi convenablement $d \dot{g}_{0}, d_{k} \dot{h}_{0}$, $1 \leq k \leq m\left(\pi_{0}\right)$, il se trouve une mesure $\nu_{0}$ sur $\hat{G}_{0}$ possédant les propriétés requises dans le théorème. Alors, nous conviendra l'image $\nu$ de $\nu_{0}$ par induction des représentations. En effet, quand on considère la désintégration de $\nu_{0}$ relative à $\nu$ :

$$
\nu_{0}=\int_{\hat{G}} \nu_{\pi} d \nu(\pi)
$$

la fibre au-dessus de $\pi$ se compose des $\pi_{0}^{j}$ pour $1 \leq j \leq s$ et le Corollaire 1 dit que $\nu_{\pi}=\sum_{j=1}^{s} c_{j} \delta_{\pi_{j}}$ avec $c_{j}>0$, où $\delta_{\pi^{\prime}}$ signifie la mesure de Dirac au point $\pi_{0}^{j} \in \hat{G}_{0}$. Soit $\phi \in \mathscr{D}(G)$. L'expression (4) appliquée à $\left\langle\pi_{0}^{j}(\phi) a_{\pi^{\prime}}^{k}, a_{\pi_{j}}^{k}\right\rangle$ et à $\left\langle\pi(\phi) a_{\pi}^{r}, a_{\pi}^{r}\right\rangle$, où $r=\sum_{i=1}^{j-1} m\left(\pi_{0}^{i}\right)+k$, et la Proposition 3 nous permettent de choisir $d \dot{g}, d_{k} \dot{h}$ de sorte qu'on ait, pour tous les indice $j, k$ et toute $\phi \in \mathscr{D}(G)$,

$$
\left\langle\pi(\phi) a_{\pi}^{r}, a_{\pi}^{r}\right\rangle=c_{j}\left\langle\pi_{0}^{j}(\phi) a_{\pi_{j}}^{k}, a_{\pi_{0}}^{k}\right\rangle .
$$

Avec ces choix on calcul: pour n'importe quelle $\phi \in \mathscr{D}(G)$,

$$
\begin{aligned}
\phi_{H}^{f}(e) & =\int_{\hat{G}_{0}} \sum_{k=1}^{m\left(\pi_{0}\right)}\left\langle\pi_{0}(\phi) a_{\pi_{0}}^{k}, a_{\pi_{0}}^{k}\right\rangle d \nu_{0}\left(\pi_{0}\right) \\
& =\int_{\hat{G}} d \nu(\pi) \int_{\hat{G}_{0}} \sum_{k=1}^{m\left(\pi_{0}\right)}\left\langle\pi_{0}(\phi) a_{\pi_{0}}^{k}, a_{\pi_{0}}^{k}\right\rangle d \nu_{\pi}\left(\pi_{0}\right) \\
& =\int_{\hat{G}} \sum_{j=1}^{s} \sum_{k=1}^{m\left(\pi_{0}^{j}\right)} c_{j}\left\langle\pi_{0}^{j}(\phi) a_{\pi_{\delta}}^{k}, a_{\pi_{0}}^{k}\right\rangle d \nu(\pi) \\
& =\int_{\hat{G}} \sum_{k=1}^{m(\pi)}\left\langle\pi(\phi) a_{\pi}^{k}, a_{\pi}^{k}\right\rangle d \nu(\pi),
\end{aligned}
$$

ce qui termine la démonstration. 
EXEMPLE 8. Considérons un cas particulier où $\mathfrak{h} \in M(f, \mathfrak{g})$. Soient $l \in G \cdot f, \mathfrak{b} \in M(l, \mathfrak{g})$ et $\pi=\operatorname{ind}_{B}^{G} \chi_{l}$ comme plus haut. Alors, $m(\pi)=1$. Les données de $d \dot{g}$ et $\dot{d h}=d_{1} \dot{h}$ nous rend capable de construire un élément $a_{\pi}=a_{\pi}^{1}$ dans $\left(\mathscr{H}_{\pi}^{-\infty}\right)^{H, \chi_{f}}$, et en réalité $\left(\mathscr{H}_{\pi}^{-\infty}\right)^{H, \chi_{f}}=\mathbf{C} a_{\pi}$. Sous réserve de la normalisation, par exemple, de $d \dot{h}$, la formule (4), combinée avec la Proposition 3, devient

$$
\left\langle\pi(\phi) a_{\pi}, a_{\pi}\right\rangle=\phi_{H}^{f}(e),
$$

ce qui est exactement notre formule de Plancherel quand on qualifie pour $\nu$ la mesure de Dirac en $\pi$.

\section{REFERENCES}

[1] L. Auslander and C. C. Moore, Unitary representations of solvable Lie groups, Mem. Amer. Math. Soc. No 62, 1966.

[2] Y. Benoist, Espaces symétriques exponentiels, Thèse de $3^{e}$ cycle, Univ. de Paris VII, 1983.

[3] Analyse harmonique sur les espaces symétriques nilpotents, J. Functional Anal., 59 (1984), 211-253.

[4] P. Bernat et al., Représentations des groupes de Lie résolubles, Dunod, Paris 1972.

[5] P. Bonnet, Transformation de Fourier des distribtuions de type positif sur un groupe de Lie unimodulaire, J. Functional Anal., 55 (1984), 220-246.

[6] F. Bruhat, Sur les représentations induites des groupes de Lie, Bull. Soc. Math. Fr., 84 (1956), 97-205.

[7] P. Cartier, Vecteurs différentiables dans les représentations unitaires des groupes de Lie, Lect. Notes in Math. 514, Springer (1975), 20-34.

[8] L. Corwin, F. P. Greenleaf and R. Penney, A general character formula for irreducible projections on $L^{2}$ of a nilmanifold, Math. Ann., 225 (1977), 21-32.

[9] V. A. Ginzburg, Symplectic geometry and representations, Functional Anal. Appl., 17 (1983), 75-76.

[10] G. Grélaud, Désintégration des représentations induites des groupes de Lie résolubles exponentiels, Thèse de $3^{e}$ cycle, Univ. de Poiters, 1973.

[11] _ Sur les représentations des groupes de Lie résolubles, Thèse, Univ. de Poitiers, 1984.

[12] R. Howe, On a connection between nilpotent groups and oscillatory integrals associated to singularities, Pacific J. Math., 73 (1977), 329-364.

[13] A. A. Kirillov, Représentations unitaires des groupes de Lie nilpotents, Uspekhi Mat. Nauk, 17 (1962), 57-110.

[14] G. Lion, Intégrale d'entrelacement sur des groupes de Lie nilpotents et indices de Maslov, Lect. Notes in Math., 587 (1977), 160-176.

[15] G. Lion et M. Vergne, The Weil Representations, Maslov Index and Theta Series, Birkhäuser, Boston 1980.

[16] C. C. Moore and J. A. Wolf, Square integrable representations of nilpotent groups, Trans. Amer. Math. Soc., 185 (1973), 445-462.

[17] R. Penney, Abstract Plancherel theorems and a Frobenius reciprocity theorem, J. Functional Anal., 18 (1975), 177-190.

[18] N. S. Poulsen, On $C^{\infty}$-vectors and intertwining bilinear forms for representations of Lie groups, J. Functional Anal., 9 (1972), 87-120. 
[19] L. Pukanszky, Leçon sur les représentations des groupes, Dunod, Paris 1967.

[20] S. R. Quint, Decomposition of induced representations of solvable exponential Lie groups, Dissertation, Univ. of California, Berkeley 1973.

[21] H. Whitney, Elementary structure of real algebraic varieties, Ann. of Math., 66 (1957), 545-556.

Received August 30, 1985 and in revised form August 4, 1986.

UNIVERSITE DE KYUSHU 33

FukuoKa, Postal No. 812

JOPAN 


\section{PACIFIC JOURNAL OF MATHEMATICS EDITORS}

\author{
V. S. VARADARAJAN \\ (Managing Editor) \\ University of California \\ Los Angeles, CA 90024 \\ HeRbert Clemens \\ University of Utah \\ Salt Lake City, UT 84112 \\ R. FINN \\ Stanford University \\ Stanford, CA 94305
}

\author{
HERMANN FLASCHKA \\ University of Arizona \\ Tucson, AZ 85721 \\ RAMESH A. GANGOLLI \\ University of Washington \\ Seattle, WA 98195 \\ VAUGHAN F. R. JONES \\ University of California \\ Berkeley, CA 94720 \\ ROBION KIRBY \\ University of California \\ Berkeley, CA 94720
}

\author{
C. C. MOORE \\ University of California \\ Berkeley, CA 94720 \\ H. SAMELSON \\ Stanford University \\ Stanford, CA 94305 \\ HAROLD STARK \\ University of California, San Diego \\ La Jolla, CA 92093
}

\section{ASSOCIATE EDITORS}
R. Arens
E. F. BECKENBACH
B. H. NEUMANN
F. WOLF
K. YosHIDA (1906-1982)

\section{SUPPORTING INSTITUTIONS}

UNIVERSITY OF ARIZONA
UNIVERSITY OF BRITISH COLUMBIA
CALIFORNIA INSTITUTE OF TECHN
UNIVERSITY OF CALIFORNIA
MONTANA STATE UNIVERSITY
UNIVERSITY OF NEVADA, RENO
NEW MEXICO STATE UNIVERSITY
OREGON STATE UNIVERSITY

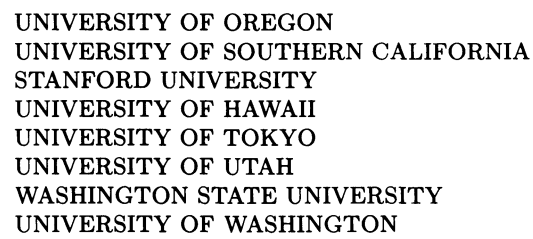

The Supporting Institutions listed above contribute to the cost of publication of this Journal, but they are not owners or publishers and have no responsibility for its content or policies.

Mathematical papers intended for publication in the Pacific Journal of Mathematics should be in typed form or offset-reproduced (not dittoed), double spaced with large margins. Please do not use built up fractions in the text of the manuscript. However, you may use them in the displayed equations. Underline Greek letters in red, German in green, and script in blue. The first paragraph must be capable of being used separately as a synopsis of the entire paper. In particular it should contain no bibliographic references. Please propose a heading for the odd numbered pages of less than 35 characters. Manuscripts, in triplicate, may be sent to any one of the editors. Please classify according to the scheme of Math. Reviews, Index to Vol. 39. Supply name and address of author to whom proofs should be sent. All other communications should be addressed to the managing editor, or Elaine Barth, University of California, Los Angeles, California 90024.

There are page-charges associated with articles appearing in the Pacific Journal of Mathematics. These charges are expected to be paid by the author's University, Government Agency or Company. If the author or authors do not have access to such Institutional support these charges are waived. Single authors will receive 50 free reprints; joint authors will receive a total of 100 free reprints. Additional copies may be obtained at cost in multiples of 50 .

The Pacific Journal of Mathematics is issued monthly as of January 1966. Regular subscription rate: $\$ 190.00$ a year (5 Vols., 10 issues). Special rate: $\$ 95.00$ a year to individual members of supporting institutions.

Subscriptions, orders for numbers issued in the last three calendar years, and changes of address should be sent to Pacific Journal of Mathematics, P.O. Box 969, Carmel Valley, CA 93924, U.S.A. Old back numbers obtainable from Kraus Periodicals Co., Route 100, Millwood, NY 10546.

The Pacific Journal of Mathematics at P.O. Box 969, Carmel Valley, CA 93924 (ISSN 0030-8730) publishes 5 volumes per year. Application to mail at Second-class postage rates is pending at Carmel Valley, California, and additional mailing offices. Postmaster: send address changes to Pacific Journal of Mathematics, P.O. Box 969, Carmel Valley, CA 93924.

PUBLISHED BY PACIFIC JOURNAL OF MATHEMATICS, A NON-PROFIT CORPORATION Copyright (C) 1987 by Pacific Journal of Mathematics 


\section{Pacific Journal of Mathematics \\ Vol. 127, No. $2 \quad$ February, 1987}

Richard Martin Aron and Robert Henry Lohman, A geometric function determined by extreme points of the unit ball of a normed space .... . . 209

Kari Astala and M. S. Ramanujan, $(s)$-nuclear sets and operators $\ldots \ldots \ldots 233$ Earl Robert Berkson, Thomas Alastair Gillespie and Paul Scott Muhly, Analyticity and spectral decompositions of $L^{p}$ for compact abelian

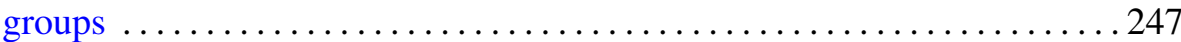

Ronald P. Brown, Real closures of fields at orderings of higher level . . . . . 261

Ronald P. Brown, The behavior of chains of orderings under field

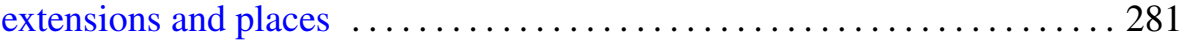

Rodney Graham Downey, Subsets of hypersimple sets .............. 299

Manfred Droste and Saharon Shelah, On the universality of systems of

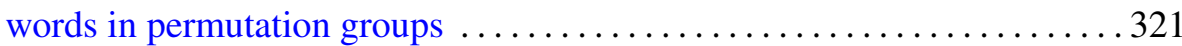

Hidenori Fujiwara, Représentations monomiales des groupes de Lie

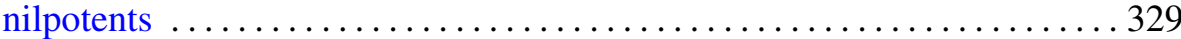

Rupert Lasser, Convolution semigroups on hypergroups $\ldots \ldots \ldots \ldots \ldots 35$

Marcus Marlene Marsh, $u$-mappings on trees $\ldots \ldots \ldots \ldots \ldots \ldots \ldots \ldots \ldots \ldots$

Peter Andrew Symonds, Localization in the classification of flat

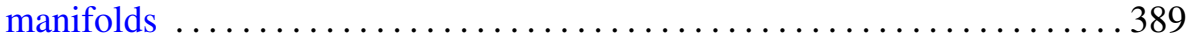

